\title{
АСИМПТОТИКА ФУНДАМЕНТАЛЬНОГО РЕШЕНИЯ ДЛЯ УРАВНЕНИЯ ДИФФУЗИИ В ПЕРИОДИЧЕСКОЙ СРЕДЕ НА БОЛЬШИХ ВРЕМЕНАХ И ЕЕ ПРИМЕНЕНИЕ К ОЦЕНКАМ ТЕОРИИ УСРЕДНЕНИЯ
}

\author{
(c) 2017 г. В. В. ЖИКОВ, С. Е. ПАСТУХОВА
}

\begin{abstract}
АннотАция. Рассматривается уравнение диффузии в бесконечной 1-периодической среде. Для фундаментального решения находятся аппроксимации при больших значениях времени $t$. Погрешность аппроксимаций имеет поточечную и интегральную оценки порядка $O\left(t^{-\frac{d+j+1}{2}}\right)$ и $O\left(t^{-\frac{j+1}{2}}\right), j=0,1, \ldots$, соответственно. Аппроксимации строятся из известного фундаментального решения усредненного уравнения, имеющего постоянные коэффициенты, и его производных, а также решений серии вспомогательных задач на ячейке периодичности. Серия задач на ячейке выписывается рекуррентным образом. Эти результаты используются для построения аппроксимаций операторной экспоненты исходного уравнения диффузии с оценками погрешности по операторным нормам в $L^{p}$-пространствах, $1 \leqslant p \leqslant \infty$. Для аналогичного уравнения в $\varepsilon$-периодической среде ( $\varepsilon$-малый параметр) получаются аппроксимации операторной экспоненты в $L^{p}$-операторных нормах при фиксированном времени с погрешностью порядка $O\left(\varepsilon^{n}\right), n=1,2, \ldots$
\end{abstract}

\section{ОГЛАВЛЕНИЕ}

1. Введение ... . . . . . . . . . . . . . . . . . . . . . . . 223

2. Блоховское представление экспоненты $e^{-t A} \ldots \ldots \ldots \ldots \ldots$. . . . . . . . 227

3. О спектре оператора $A(k) \ldots \ldots$. . . . . . . . . . . . . . . . . . . . 229

4. Выделение главного члена асимптотики . . . . . . . . . . . . . . . . . . . . . . . . . . 232

5. Вспомогательные задачи на ячейке . . . . . . . . . . . . . . . . . . . . . . . . 235

6. Полное аналитическое разложение . . . . . . . . . . . . . . . . . . . . . 237

7. Приближения высокого порядка . . . . . . . . . . . . . . . . . . . . . . . . . 238

8. Уравнения с $\varepsilon$-периодическими коэффициентами . . . . . . . . . . . . . 241

9. Доказательство леммы $7.1 \ldots \ldots$. . . . . . . . . . . . . . . . . . . . 243

Список литературы . . . . . . . . . . . . . . . . . . . . . . . . . . 243

\section{1. ВВЕДЕНИЕ}

1.1. Рассмотрим задачу Коши для функции $u=u(x, t), x \in \mathbb{R}^{d}, t \geqslant 0$ :

$$
\left\{\begin{array}{l}
\frac{\partial u}{\partial t}-\operatorname{div}(a(x) \nabla u)=0, t>0, \\
\left.u\right|_{t=0}=f \in C_{0}^{\infty}\left(\mathbb{R}^{d}\right)
\end{array}\right.
$$

с измеримой вещественной симметрической матрицей $a(x)$. Предполагается, что матрица $a(x)$ равномерно эллиптична, т. е.

$$
\nu \xi^{2} \leqslant a(x) \xi \cdot \xi \leqslant \nu^{-1} \xi^{2} \quad \forall \xi \in \mathbb{R}^{d}, \nu>0 .
$$

Имеем уравнение диффузии в неоднородной среде, $a(x)$ - матрица диффузии.

Работа выполнена при финансовой поддержке Российского фонда фундаментальных исследований (проект 14-0100192 А), а также Министерства образования РФ (задание № 1.3270.2017/ПЧ) 
Задачу (1.1) перепишем в виде

$$
\left\{\begin{array}{l}
\frac{\partial u}{\partial t}+A u=0, t>0, \\
\left.u\right|_{t=0}=f
\end{array}\right.
$$

где $A$ - оператор в $L^{2}\left(\mathbb{R}^{d}\right)$, заданный квадратичной формой

$$
\int_{\mathbb{R}^{d}} a \nabla u \cdot \nabla u d x \text { на } H^{1}\left(\mathbb{R}^{d}\right) .
$$

Квадратичная форма является замкнутой, а сам оператор $A$-неотрицательным и самосопряженным. Решение задачи Коши (1.1) запишется как

$$
u(\cdot, t)=e^{-t A} f .
$$

Предположим также, что матрица $a(x)$ периодична по каждому переменному $x_{1}, \ldots x_{d}$ с периодом 1 , единичный куб $\square=[-1 / 2,1 / 2)^{d}$ есть ячейка периодичности. Тогда известно асимптотическое поведение или асимптотическое представление решения $u=u(x, t)$ при $t \rightarrow \infty$, иными словами, поведение или представление полугруппы $e^{-t A}$ при большом значении времени.

Оказывается, что на больших временах за поведение полугруппы отвечает постоянная усредненная матрица диффузии $a^{0}$ (процедура ее отыскания по исходной матрице $a(x)$ указана ниже в $(1.10),(1.11))$ и надо рассматривать усредненную задачу

$$
\left\{\begin{array}{l}
\frac{\partial u^{0}}{\partial t}+A_{0} u^{0}=0, t>0 \\
\left.u^{0}\right|_{t=0}=f \in C_{0}^{\infty}\left(\mathbb{R}^{d}\right)
\end{array}\right.
$$

с оператором диффузии

$$
A_{0}=-\operatorname{div}\left(a^{0} \nabla\right),
$$

существенно более простым, чем исходный, хотя той же структуры. Например, в [7] было доказано предельное соотношение

$$
\lim _{t \rightarrow \infty} \sup _{\|f\|_{L^{\infty}\left(\mathbb{R}^{d}\right)} \leqslant 1}\left\|u(x, t)-u^{0}(x, t)\right\|_{L^{\infty}\left(\mathbb{R}^{d}\right)}=0,
$$

означающее сходимость полугруппы по операторной норме в $L^{\infty}\left(\mathbb{R}^{d}\right)$, т. е.

$$
\lim _{t \rightarrow \infty}\left\|e^{-t A}-e^{-t A_{0}}\right\|_{L^{\infty}\left(\mathbb{R}^{d}\right) \rightarrow L^{\infty}\left(\mathbb{R}^{d}\right)}=0 .
$$

Позже в [8] установлена оценка скорости этой сходимости по времени

$$
\left\|e^{-t A}-e^{-t A_{0}}\right\|_{L^{\infty}\left(\mathbb{R}^{d}\right) \rightarrow L^{\infty}\left(\mathbb{R}^{d}\right)} \leqslant \frac{c}{\sqrt{t}}
$$

с константой, зависящей лишь от размерности $d$ и постоянной эллиптичности $\nu$.

Была доказана также оценка

$$
\left\|e^{-t A}-e^{-t A_{0}}\right\|_{L^{2}\left(\mathbb{R}^{d}\right) \rightarrow L^{2}\left(\mathbb{R}^{d}\right)} \leqslant \frac{c}{\sqrt{t}}
$$

с константой того же типа, что в $(1.4)$, см. [17,23].

Наконец, недавно в работе [10] установлена оценка

$$
\left\|e^{-t A} f-e^{-t A_{0}} f\right\|_{L^{p}\left(\mathbb{R}^{d}\right)} \leqslant \frac{c}{\sqrt{t}}\|f\|_{L^{p}\left(\mathbb{R}^{d}\right)} \quad \forall p \in[1, \infty]
$$

с единой константой для всех $p$. Предыдущие оценки (1.4) и (1.5) вытекают отсюда при $p=\infty$ и $p=2$, для вероятностной интерпретации уравнения диффузии особенно важен случай $p=1$.

Часто операторные оценки можно вывести из оценок поточечного характера. Примером операторной оценки служит (1.5). Примером поточечной оценки является

$$
\left|K(x, y, t)-K_{0}(x, y, t)\right| \leqslant \frac{c}{t^{\frac{d+1}{2}}}, \quad x, y \in \mathbb{R}^{d}, \quad c=\operatorname{const}(d, \nu) .
$$


Здесь $K$ - фундаментальное решение для параболического уравнения $\frac{\partial u}{\partial t}+A u=0$, иными словами, $K$ - ядро интегрального оператора $e^{-t A} ; K_{0}$ - аналогичный объект для усредненного уравнения, который ввиду постоянства коэффициентов уравнения можно точно найти с помощью преобразования Фурье, а именно,

$$
K_{0}(x, y, t)=(4 \pi t)^{-\frac{d}{2}}\left(\operatorname{det} a^{0}\right)^{-\frac{1}{2}} e^{-\frac{\left(a^{0}\right)^{-1}(x-y) \cdot(x-y)}{4 t}} .
$$

Из поточечной оценки (1.7) с помощью общих соображений (экспоненциальная оценка НэшаАронсона) получается интегральная оценка

$$
\int_{\mathbb{R}^{d}}\left|K(x, y, t)-K_{0}(x, y, t)\right| d y \leqslant \frac{c}{\sqrt{t}} \quad \forall x \in \mathbb{R}^{d},
$$

которая и играет основную роль. Дело в том, что из нее (на основе стандартных фактов об оценке нормы интегрального оператора, см. ниже лемму 1.1) немедленно получается $L^{p}$-оценка (1.6). Интегральная оценка (1.9) доказана в работе В. В. Жикова [8] 1989 года (см. также более подробное доказательство ее в недавней работе [10]). В настоящей работе доказываются более точные интегральные оценки, которые отвечают не нулевому, а следующим приближениям для фундаментального решения $K$. Основные результаты сформулированы в разделах 4,7 и 8 .

Для доказательства наших результатов используем предложенную в [8] версию спектрального метода, в основе которой лежит блоховское разложение функций и блоховское представление операторной экспоненты, точнее, ее ядра как интегрального оператора.

В целом спектральный подход к асимптотическим задачам на периодических структурах имеет давнюю историю и появился прежде всего в физической литературе, оставаясь долгое время без строгого математического обоснования. В теории усреднения интерес к спектральному методу возник с самого начала (см. [19]), причем он периодически затухал и возобновлялся. Вышло большое количество работ в этом направлении. Отметим здесь только одни из наиболее ранних применений спектрального метода в теории усреднения - работы $[2,16]$, а также интересные публикации $[1,3,20]$, имеющие отношение к параболическим уравнениям. В последние полтора десятилетия с появлением работы [4] наблюдается повышенный интерес к спектральному подходу в связи с применением его к операторным оценкам усреднения.

1.2. Приведем формулы для определения усредненной матрицы диффузии:

$$
a^{0}=\langle a(\cdot)(I+\nabla N(\cdot)\rangle
$$

где $I$ - единичная матрица, вектор $N(x)=\left(N_{1}(x), \ldots, N_{d}(x)\right)$ составлен из решений задач на ячейке

$$
\begin{gathered}
N_{j} \in H_{\mathrm{per}}^{1}(\square), \quad \operatorname{div}\left(a(x)\left(\nabla N_{j}+e_{j}\right)\right)=0, \quad\left\langle N_{j}\right\rangle=0, \\
j=1, \ldots, d .
\end{gathered}
$$

Здесь и всюду далее $e_{1}, \ldots, e_{d}-$ канонический базис в $\mathbb{R}^{d}, H_{\mathrm{per}}^{1}(\square)$ - пространство Соболева 1 периодических функций,

$$
\langle\cdot\rangle=\int_{\square} \cdot d x-\text { среднее по ячейке. }
$$

Решение задачи (1.11) понимается в смысле интегрального тождества

$$
\int_{\square} a\left(e_{j}+\nabla N_{j}\right) \cdot \nabla \varphi d x=0 \quad \forall \varphi \in C_{p e r}^{\infty}(\square) .
$$

Задача (1.11) представляет собой частный случай общей задачи на ячейке

$$
v \in H_{\mathrm{per}}^{1}(\square), \quad \operatorname{div}(a(x) \nabla v)=F_{0}+\operatorname{div} F,
$$

где $F_{0} \in L^{2}(\square)$ и $F \in L^{2}(\square)^{d}$. Ядро этой задачи составляют константы, следовательно, условие ее разрешимости есть условие ортогональности $F_{0} \perp 1$ в $L^{2}(\square)$, т. е. $\left\langle F_{0}\right\rangle=0$. В случае (1.11) это условие разрешимости, очевидно, выполнено.

Важной особенностью задачи на ячейке (1.11) является ограниченность решения: $N_{j} \in L^{\infty}(\square)$ в силу обобщенного принципа максимума (см. [12, приложение В к гл. II]). 
В теории усреднения хорошо известно, что матрица (1.10) симметрична и удовлетворяет оценке

$$
a^{0} k \cdot k \geqslant \nu k^{2} \quad \forall k \in \mathbb{R}^{d}
$$

с той же константой, что в (1.2) (см., например, [9]).

1.3. До сих пор мы оперировали нулевым приближением для $K$. Спектральный метод позволяет дать полное асимптотическое разложение $K$. Пока ограничимся лишь первым приближением. Используя решения задачи на ячейке (1.11), определим первое приближение равенством

$$
K_{1}(x, y, t)=K_{0}(x, y, t)+\left(N_{j}(x)-N_{j}(y)\right) \frac{\partial}{\partial x_{j}} K_{0}(x, y, t) .
$$

(Ядро $K_{1}$ симметрично в виду кососимметричности $\frac{\partial}{\partial x_{j}} K_{0}$.) Справедливы оценки

$$
\begin{gathered}
\left|K(x, y, t)-K_{1}(x, y, t)\right| \leqslant \frac{c}{t^{\frac{d+2}{2}}}, \\
\int_{\mathbb{R}^{d}}\left|K(x, y, t)-K_{1}(x, y, t)\right| d y \leqslant \frac{c}{t}
\end{gathered}
$$

с константами того же типа, что в (1.7) и (1.9).

1.4. Поточечные оценки (1.7) и (1.15) позволяют сделать некоторые выводы вероятностного характера, относящиеся к центральной предельной теореме. Пусть $\xi^{t}$ обозначает случайную величину с плотностью распределения $K(x, y, t)$ ( $y$ - параметр, указывающий локализацию случайной величины в начальный момент). Тогда случайная величина $\frac{\xi^{t}-y}{\sqrt{t}}$ имеет плотность распределения

$$
p_{y}(x, t)=t^{\frac{d}{2}} K(\sqrt{t} x+y, y, t)
$$

Тогда из (1.7) следует, что

$$
\left|p_{y}(x, t)-K_{0}(x, 0,1)\right| \leqslant \frac{c}{\sqrt{t}}
$$

равномерно по $x, y \in \mathbb{R}^{d}$.

Очевидно, что $K_{0}(x, 0,1)$ - это плотность нормального распределения с нулевым математическим ожиданием и ковариационной матрицей $2 a^{0}$. Само неравенство (1.18) есть аналог известной оценки Берри-Эссена, см. [18, с. 608].

Согласно (1.15), получаем более точное приближение для плотности распределения случайной величины $\frac{\xi^{t}-y}{\sqrt{t}}$, а именно,

$$
\left|p_{y}(x, t)-K_{0}(x, 0,1)-\frac{1}{\sqrt{t}}\left(N_{j}(\sqrt{t} x+y)-N_{j}(y)\right) \frac{\partial}{\partial x_{j}} K_{0}(x, 0,1)\right| \leqslant \frac{c}{t}
$$

равномерно по $x, y \in \mathbb{R}^{d}$.

1.5. Приведем точную формулировку ключевого для нас результата о норме интегрального оператора, который часто называют леммой Шура.

Лемма 1.1. Пусть $S(x, y)$-симметрическое непрерьвное на $\mathbb{R}^{d} \times \mathbb{R}^{d}$ ядро

$$
\int_{\mathbb{R}^{d}}|S(x, y)| d y \leqslant c \forall x \in \mathbb{R}^{d} .
$$

Тогда для

$$
u(x)=\int_{\mathbb{R}^{d}} S(x, y) f(y) d y
$$

выполнено неравенство

$$
\|u\|_{L^{p}\left(\mathbb{R}^{d}\right)} \leqslant c\|f\|_{L^{p}\left(\mathbb{R}^{d}\right)} \quad \forall p \in[1, \infty]
$$


Доказательство. Считаем для простоты записи, что $S(x, y) \geqslant 0$. Тогда

$$
\begin{gathered}
|u(x)| \leqslant \int_{\mathbb{R}^{d}}|S(x, y) f(y)| d y, \\
\int_{\mathbb{R}^{d}}|u(x)| d x \leqslant \int_{\mathbb{R}^{d}}|f(y)| d y \int_{\mathbb{R}^{d}} S(x, y) d x \leqslant c \int_{\mathbb{R}^{d}}|f(y)| d y .
\end{gathered}
$$

Оценка для $p=1$ доказана.

При $p>1, q=\frac{p}{p-1}$, имеем по неравенству Гельдера

$$
\begin{gathered}
|u(x)|^{p} \leqslant\left|\int_{\mathbb{R}^{d}} S^{\frac{1}{q}}(x, y) S^{\frac{1}{p}}(x, y) f(y) d y\right|^{p} \leqslant \\
\left(\int_{\mathbb{R}^{d}} S(x, y) d y\right)^{\frac{p}{q}} \int_{\mathbb{R}^{d}} S(x, y)|f(y)|^{p} d y \leqslant c^{\frac{p}{q}} \int_{\mathbb{R}^{d}} S(x, y)|f(y)|^{p} d y, \\
\int_{\mathbb{R}^{d}}|u(x)|^{p} d x \leqslant c^{\frac{p}{q}+1} \int_{\mathbb{R}^{d}}|f(y)|^{p} d y .
\end{gathered}
$$

Отсюда следует оценка (1.21). Лемма доказана.

Общее изложение леммы Шура см. [13, гл. I, теорема 4.8].

\section{2. БЛОХОВСКОЕ ПРЕДСТАВЛЕНИЕ ЭКСПОНЕНТЫ $e^{-t A}$}

Основную роль в наших построениях будет играть представление решения задачи Коши (1.1) через решения некоторых периодических задач - так называемое блоховское разложение (в определенном смысле это аналог разложения в ряд Фурье для периодической функции). Отсюда получается представление экспоненты $e^{-t A}$, действующей в $L^{2}\left(\mathbb{R}^{d}\right)$, через операторные экспоненты, действующие в $L^{2}(\square)$.

2.1. Напомним известное преобразование Блоха-Гельфанда. Для $f \in C_{0}^{\infty}\left(\mathbb{R}^{d}\right)$ положим

$$
U f=\hat{f}(x, k)=\frac{1}{(2 \pi)^{\frac{d}{2}}} \sum_{h \in Z^{d}} e^{-i k \cdot(x+h)} f(x+h),
$$

где сумма конечна при каждом фиксированном $x$. Заметим, что

1. функция $\hat{f}(x, k)$ является 1-периодической по $x_{1}, \ldots x_{d}$;

2. функция $e^{i k \cdot x} \hat{f}(x, k)$ является $2 \pi$-периодической по $k_{1}, \ldots k_{d}$; ячейками периодичности будут $\square=[-1 / 2,1 / 2)^{d}$ и $\square^{*}=[-\pi, \pi)^{d}$ соответственно.

Для преобразования (2.1) справедлива формула обращения

$$
f(x)=\frac{1}{(2 \pi)^{\frac{d}{2}}} \int_{\square^{*}} e^{i k \cdot x} \hat{f}(x, k) d k .
$$

Кроме того,

$$
\int_{\mathbb{R}^{d}}|f(x)|^{2} d x=\int_{\square \times \square^{*}}|\hat{f}(x, k)|^{2} d x d k
$$

и преобразование $U$ может быть расширено (по непрерывности) до унитарного оператора

$$
U: L^{2}\left(\mathbb{R}^{d}\right) \rightarrow L^{2}\left(\square \times \square^{*}\right) .
$$

Строгое построение оператора $U$ дано в [24].

Обратный оператор $U^{-1}: L^{2}\left(\square \times \square^{*}\right) \rightarrow L^{2}\left(\mathbb{R}^{d}\right)$ задается равенством (2.2) для любых $\hat{f} \in$ $L^{2}\left(\square \times \square^{*}\right)$. В то же время определение (2.1) непосредственно применимо, если $f$ достаточно быстро убывает при $|x| \rightarrow \infty$, например, если $e^{s|x|} f(x) \in L^{2}\left(\mathbb{R}^{d}\right)$ для некоторого $s>0$. Решение 
задачи Коши (1.1) удовлетворяет такому условию, поскольку начальное значение $f \in C_{0}^{\infty}\left(\mathbb{R}^{d}\right)$ (доказательство этого факта дано в [9, гл. II]).

2.2. С периодической матрицей $a(x)$ свяжем семейство операторов

$$
\begin{gathered}
A(k)=e^{-i k \cdot x} A e^{i k \cdot x}=-e^{-i k \cdot x} \operatorname{div}\left(a(x) \nabla e^{i k \cdot x}\right)= \\
-(\nabla+i k)^{*} a(\nabla+i k)=-\operatorname{div} a \nabla-i \operatorname{div} a k-i k \cdot a \nabla+a k \cdot k,
\end{gathered}
$$

в которое вкладывается исходный оператор $A(0)=A$, где $i=\sqrt{-1}$, а вещественный параметр $k \in \mathbb{R}^{d}$ называется квазиимпульсом.

Строго говоря, оператор $A(k)$ задается в пространстве $L^{2}(\square)$ (комплекснозначных функций) квадратичной формой

$$
\int_{\square} a(x)(\nabla u+i k u) \cdot(\nabla \bar{u}-i k \bar{u}) d x \text { на } H_{p e r}^{1}(\square) \subset L^{2}(\square),
$$

где черта сверху означает комплексное сопряжение. Квадратичная форма, очевидно, замкнута, а сам оператор - неотрицательный и самосопряженный.

Для функции

$$
v(x, t)=u(x, t) e^{-i k \cdot x},
$$

где $u(x, t)$ - решение задачи Коши (1.1), очевидно, выполнены соотношения

$$
\left\{\begin{array}{l}
\frac{\partial v}{\partial t}-A(k) v=0, t>0, \\
\left.v\right|_{t=0}=e^{-i k \cdot x} f(x) .
\end{array}\right.
$$

Отсюда сдвигом на целочисленные векторы и суммированием с учетом периодичности матрицы $a(x)$ выводим, что полученная из $u(x, t)$ по формуле $(2.1)$ функция $\hat{u}(x, k, t)$ периодична по $x$ и $\hat{u}(\cdot, k, \cdot)$ есть решение задачи Коши на ячейке

$$
\left\{\begin{array}{l}
\frac{\partial \hat{u}}{\partial t}-A(k) \hat{u}=0, t>0 \\
\left.\hat{u}\right|_{t=0}=\hat{f}(x, k)
\end{array}\right.
$$

где $k$ выступает параметром. Согласно (2.2) можно вернуться к исходному решению

$$
u(x, t)=\frac{1}{(2 \pi)^{\frac{d}{2}}} \int_{\square^{*}} e^{i k \cdot x} \hat{u}(x, k, t) d k .
$$

Таким образом, получено искомое представление решения $u(x, t)$ исходной задачи (1.1) через решения $\hat{u}(x, k, t)$ периодических задач (2.5). Запишем это как

$$
e^{-t A} f=(2 \pi)^{-\frac{d}{2}} \int_{\square^{*}} e^{i k \cdot x} e^{-t A(k)} \hat{f}(x, k) d k,
$$

откуда

$$
e^{-t A}=U^{-1} e^{-t \hat{A}} U
$$

где полугруппа $e^{-t \hat{A}}$ действует в $L^{2}\left(\square \times \square^{*}\right)=L^{2}\left(\square^{*}, L^{2}(\square)\right)$ «послойно» по $k$,

$$
e^{-t \hat{A}} \hat{f}=e^{-t A(k)} \hat{f}(\cdot, k) .
$$

Отметим один общий факт. Если $B=B(k)$ - ограниченный в $L^{2}(\square)$ оператор, непрерывно (по операторной норме) зависящий от $k \in \square^{*}$, то послойное его действие в $L^{2}\left(\square \times \square^{*}\right)=L^{2}\left(\square^{*}, L^{2}(\square)\right)$ определяется равенством

$$
\hat{B} f=B(k) f(\cdot, k) \text { для } f \in L^{2}\left(\square \times \square^{*}\right)=L^{2}\left(\square^{*}, L^{2}(\square)\right) .
$$

При этом очевидна оценка

$$
\|\hat{B}\| \leqslant \sup _{k \in \square^{*}}\|B(k)\|
$$

для соответствующих операторных норм, стоящих в левой и правой частях. 
Представление (2.7) для полугруппы $e^{-t A}$, называемое блоховским, есть основной итог этого раздела. Дальнейшая цель - изучить полугруппу $e^{-t A(k)}$, связанную с исходной полугруппой $e^{-t A}$ формулами $(2.7),(2.8)$. Естественно начать с изучения спектра оператора $A(k)$.

\section{O CПЕКTPE OПЕРATOPA $A(k)$}

3.1. Свойство разделения спектра. При каждом $k \in \mathbb{R}^{d}$ имеем неотрицательный самосопряженный в $L^{2}(\square)$ оператор $A(k)$ (см. (2.4)), резольвента его компактна. Спектр оператора $A(k)$ представляет собой стремящуюся к $+\infty$ последовательность собственных значений, которые располагаем по возрастанию с учетом кратности:

$$
0 \leqslant \lambda_{0}(k) \leqslant \lambda_{1}(k) \leqslant \ldots,
$$

соответствующие собственные функции

$$
\varphi_{0}(x, k), \varphi_{1}(x, k), \ldots
$$

можно выбрать так, что они образуют ортонормированный базис в $L^{2}(\square)$.

Принято называть $\lambda_{n}(k), n=0,1, \ldots$, зонными функциями. Это - непрерывные $2 \pi$-периодические функции, их можно считать определенными на двойственной ячейке $\square^{*}=[-\pi, \pi)^{d}$. Соответствующие собственные функции $\varphi_{n}(x, k)$ измеримы по $x \in \square$ и, по крайней мере, непрерывны по $k \in \square^{*}$. Анализ, проведенный далее в пункте 4.2 , показывает, что $\varphi_{n}(x, k)$ - ограниченные функции.

Начнем с описания спектра невозмущенного оператора $A=A(0)$, которое легко получить благодаря неравенству Пуанкаре

$$
\int_{\square}|v|^{2} d x \leqslant c_{P} \int_{\square}|\nabla v|^{2} d x \quad \forall v \in H^{1}(\square), \quad\langle v\rangle=0 .
$$

Здесь функция $v$ не обязательно периодична.

Лемма 3.1. Oператор А имеет простое собственное значение $\lambda_{0}=\lambda_{0}(0)=0$ с собственной функцией $\varphi_{0}(x) \equiv 1$, а другие собственные значения $\lambda_{j}=\lambda_{j}(0), j \geqslant 1$, лежат правее точки $c_{1}=$ $c_{P}^{-1} \nu$, где $c_{P}-$ константа из неравенства Пуанкаре, $\nu$-константа эллиптичности из (1.2).

Рассмотрим возмущенный оператор $A(k), k \in \square^{*}$. Для изучения спектра $S p A(k)$ введем оператор сравнения $J(k)$. Он получается из $A(k)$, если взять $a(x)=I$, другими словами,

$$
J(k)=-e^{-i k \cdot x} \Delta e^{i k \cdot x}=-\Delta-2 i k \nabla+k^{2} .
$$

Собственными функциями и собственными значениями оператора $J(k)$ будут

$$
e^{i 2 \pi n x},(2 \pi n+k)^{2}, n \in \mathbb{Z}^{d}, k \in \square^{*} .
$$

Легко видеть, что

$$
\min _{k \in \square^{*}}(2 \pi n+k)^{2} \geqslant \pi^{2}
$$

для $\underset{\pi}{\forall n} \in \mathbb{Z}^{d}, n \neq 0$. Поэтому наименьшее собственное значение $k^{2}$ (при $n=0$ ) простое при $|k| \leqslant \frac{\pi}{2}$.

Из неравенства $\nu I \leqslant a(x) \leqslant \nu^{-1} I$ имеем

$$
\nu J(k) \leqslant A(k) \leqslant \nu^{-1} J(k) .
$$

Отсюда по принципу минимакса выводим интересующие нас свойства собственных значений оператора $A(k)$ :

1. выполнена оценка

$$
\nu k^{2} \leqslant \lambda_{0}(k) \leqslant \nu^{-1} k^{2}, \quad k \in \square^{*} ;
$$

2. $\lambda_{0}(k)-$ простое при $|k| \leqslant r_{0}=\frac{\nu \pi}{2}$;

3. $\lambda_{1}(k) \geqslant \delta_{0}=\nu \pi^{2} \forall k \in \square^{*}$.

В частности, доказана 


\section{Лемма 3.2.}

(i) На интервале $\left[0, \frac{\delta_{0}}{2}\right]$ лежит единственное собственное значение $\lambda_{0}(k)$, если $|k| \leqslant r_{0}$, где $\delta_{0}$ и $r_{0}$ указаны вынше.

(ii) Если $|k| \geqslant \tau>0$, то весь спектр оператора $A(k)$ лежит правее некоторой точки $r(\tau)>0$.

Теперь отметим свойство четности, заключающееся в том, что верны следующие равенства при достаточно малых $|k|$ (например, при $|k| \leqslant r_{0}$ ):

$$
\lambda_{0}(-k)=\lambda_{0}(k), \quad \varphi_{0}(x,-k)=\overline{\varphi_{0}(x, k)} .
$$

Эти равенства легко установить, исходя из того, что собственное значение $\lambda_{0}(k)$ простое и вещественное, а кроме того, $\overline{A(k)}=A(-k)$. В самом деле, для нормированной собственной функции $\varphi_{0}(x, k)$ справедливы соотношения

$$
\begin{aligned}
A(k) \varphi_{0}(x, k)= & \lambda_{0}(k) \varphi_{0}(x, k) \Rightarrow \overline{A(k) \varphi_{0}(x, k)}=\overline{\lambda_{0}(k) \varphi_{0}(x, k)} \Rightarrow \\
& A(-k) \overline{\varphi_{0}(x, k)}=\lambda_{0}(k) \overline{\varphi_{0}(x, k)},
\end{aligned}
$$

т. е. $\overline{\varphi_{0}(x, k)}$ - нормированная собственная функция оператора $A(-k)$ с собственным значением $\lambda_{0}(k)$. Но в силу свойства разделения спектра (см. лемму $\left.3.2,(i)\right) \lambda_{0}(-k)$-единственное собственное значение (притом простое) оператора $A(-k)$ на интервале $\left[0, \frac{\delta_{0}}{2}\right]$, а $\varphi_{0}(x,-k)-$ соответствующая нормированная собственная функция. Отсюда вытекают оба равенства (3.5).

По теории возмущений собственное значение $\lambda_{0}(k)$ аналитично по $k$ в достаточно малой окрестности точки $k=0$, т. е. при $|k| \leqslant r_{1}$. Ввиду четности $\lambda_{0}(k)$ тейлоровское разложение $\lambda_{0}(k)$ в точке $k=0$ до четвертого порядка имеет вид

$$
\lambda_{0}(k)=a^{0} k \cdot k+O\left(k^{4}\right),
$$

где $a^{0}-$ некоторая симметрическая положительно определенная матрица.

Проектор $P_{0}(k)$ на одномерное собственное пространство также аналитичен, и можно выбрать аналитическую собственную функцию $\varphi_{0}(x, k)=P_{0}(k) 1$. Более подробные рассуждения, показывающие аналитичность $\lambda_{0}(k)$ и $\varphi_{0}(x, k)$, даны в следующем пункте.

3.2. Свойство аналитичности. Исследуем сначала аналитические свойства резольвенты $(A(k)-\xi)^{-1}$ как функции параметра $k \in \mathbb{R}^{d}, \xi-$ комплексный параметр. Запишем

$$
\begin{gathered}
A(k)=A(0)+B(k), \quad A(0)=A, \\
B(k)=-i \operatorname{div} a k-i k \cdot a \nabla+a k \cdot k
\end{gathered}
$$

и положим

$$
R_{0}=(A(0)-\xi)^{-1}
$$

Тогда имеем равенство

$$
(A(k)-\xi)^{-1}=R_{0}\left(I+B(k) R_{0}\right)^{-1} .
$$

Поскольку резольвента $R_{0}$ - ограниченный оператор из $L^{2}(\square)$ в $H_{p e r}^{1}(\square)$, то оператор $B(k) R_{0}$ ограниченно действует из $L^{2}(\square)$ в $L^{2}(\square)$, причем

$$
\left\|B(k) R_{0}\right\| \leqslant \frac{1}{2} \text { при }|k| \leqslant r_{1} .
$$

Выбор числа $r_{1}>0$, очевидно, зависит от нормы оператора $R_{0}=R_{0}(\xi)$. Заметим, что $B(k) R_{0}$ есть многочлен по $k$, коэффициенты которого суть ограниченные операторы. Из (3.7), (3.8) имеем сходящийся по операторной норме ряд

$$
(A(k)-\xi)^{-1}=R_{0}+\sum_{l=1}^{+\infty}(-1)^{l} R_{0}\left(B(k) R_{0}\right)^{l} .
$$

Отсюда следует аналитичность резольвенты $(A(k)-\xi)^{-1}$ как функции аргумента $k$ в окрестности точки $k=0$. При этом в качестве $\xi$ может выступать любая точка из резольвентного множества оператора $A(0)$. 
Пусть Г- окружность в комплексной $\xi$-плоскости с центром в нуле, не содержащая внутри себя других собственных значений оператора $A(0)$, кроме нулевого (см. лемму 3.1 ). Можно проинтегрировать (3.9) по контуру Г и получить проектор

$$
P(k)=-\frac{1}{2 \pi i} \int_{\Gamma}(A(k)-\xi)^{-1} d \xi=P(0)-\frac{1}{2 \pi i} \sum_{l=1}^{+\infty} \int_{\Gamma}(-1)^{l} R_{0}\left(B(k) R_{0}\right)^{l} d \xi
$$

При достаточно малых $|k|$ верно неравенство $\|P(k)-P(0)\| \leqslant \frac{1}{2}$, из которого следует, что образ оператора $P(k)$ изоморфен образу оператора $P(0)$, в частности, оба образа одномерны. Это означает, что внутри $\Gamma$ находится в точности одно собственное значение $\lambda_{0}(k)$ и оно простое. Таким образом, еще раз другим способом доказано свойство разделения спектра оператора $A(k)$ при малых $|k|$.

Главный вывод для нас из разложения (3.10) заключается в том, что $P(k)$ - аналитическая функция. Отсюда стандартными рассуждениями (см., например, [11, гл. IV]) выводим аналитичность собственного значения $\lambda_{0}(k)$, а также собственной функции $\varphi_{0}(x, k) \in\{c P(k) 1, c \neq 0\}$. Нормируем $\varphi_{0}(x, k)$ не совсем обычным условием

$$
\left\langle\varphi_{0}(\cdot, k)\right\rangle=1
$$

(среднее по ячейке периодичности равно единице).

3.3. Идентификация первых коэффициентов тейлоровского разложения. Наряду с (3.6) запишем разложение для собственной функции

$$
\varphi_{0}(k, x)=1+c_{j}(x) k_{j}+g_{j e}(x) k_{j} k_{e}+O\left(|k|^{3}\right),
$$

$c_{j}(x), g_{j l}(x)-$ функции из $L^{2}(\square)$, более точно из $H_{\mathrm{per}}^{1}(\square)$, с нулевым средним по ячейке. Последнее согласуется с условием нормировки (3.11). Тогда

и с учетом (3.6)

$$
\begin{gathered}
-A(k) \varphi_{0}=\operatorname{div}\left(a \nabla \varphi_{0}\right)+i k_{r} a_{r s} \frac{\partial}{\partial x_{s}} \varphi_{0}+i k_{s} \frac{\partial}{\partial x_{r}}\left(a_{s r} \varphi_{0}\right)-k_{r} k_{s} a_{r s} \varphi_{0}= \\
=k_{j} \operatorname{div}\left(a \nabla c_{j}\right)+i k_{s} \frac{\partial}{\partial x_{r}} a_{r s}-k_{r} k_{s} a_{r s}+i k_{r} k_{j} a_{r s} \frac{\partial}{\partial x_{s}} c_{j}+ \\
i k_{s} k_{j} \frac{\partial}{\partial x_{r}}\left(a_{r s} c_{j}\right)+k_{j} k_{l} \operatorname{div}\left(a \nabla g_{j l}\right)+O\left(k^{3}\right)
\end{gathered}
$$

Теперь в равенстве

$$
\lambda_{0}(k) \varphi_{0}(k, x)=a^{0} k \cdot k+O\left(k^{3}\right)
$$

$$
A(k) \varphi_{0}=\lambda(k) \varphi_{0}
$$

сравним слагаемые с одинаковой степенью $k$. Сравнение линейных по $k$ слагаемых дает

$$
\operatorname{div}\left(a \nabla c_{j}\right)=-i \frac{\partial}{\partial x_{r}} a_{r j}
$$

Видим, что $c_{j}(x)$ выражается через решение $N_{j}(x)$ задачи на ячейке (см. $\left.(1.11)\right)$,

$$
c_{j}=i N_{j},
$$

поскольку согласно (1.11)

$$
\operatorname{div}\left(a \nabla N_{j}\right)=-\frac{\partial}{\partial x_{r}} a_{r j}
$$

Сравнивая слагаемые порядка $k^{2}$, с учетом равенства $c_{j}=i N_{j}$ выводим

$$
\operatorname{div}\left(a \nabla g_{j l}\right)=a_{j l}+a_{j s} \frac{\partial}{\partial x_{s}} N_{l}+\frac{\partial}{\partial x_{s}}\left(a_{j s} N_{l}\right)-a_{j l}^{0} .
$$

Относительно $g_{j l}$ получена периодическая задача вида $(1.12)$, необходимое условие разрешимости которой приводит к соотношениям

$$
a_{j l}^{0}=\left\langle a_{j l}\right\rangle+\left\langle a_{j s} \frac{\partial}{\partial x_{s}} N_{l}\right\rangle, 1 \leqslant j, l \leqslant d .
$$


Видно, что матрица $a^{0}=\left\{a_{j l}^{0}\right\}_{j, l}$ совпадает с усредненной матрицей, определенной в (1.10).

Таким образом, доказаны разложения

$$
\begin{gathered}
\lambda_{0}(k)=a^{0} k \cdot k+O\left(k^{4}\right), \\
\varphi_{0}(x, k)=1+i N_{j}(x) k_{j}+O\left(k^{2}\right),
\end{gathered}
$$

где матрица $a^{0}$ и функции $N_{j}(x)-$ те же, что в $(1.10),(1.11)$.

Собственная функция $\varphi_{0}(\cdot, k)$ оказывается не нормированной в $L^{2}(\square)$. Но после нормировки второе соотношение в (3.12) не меняется. В самом деле, поскольку $\left\langle N_{j}\right\rangle=0$ (см. (1.11)), то

$$
\begin{gathered}
\nu(k) \equiv\left\langle\varphi_{0}(\cdot, k) \overline{\varphi_{0}(\cdot, k)}\right\rangle=\left\langle 1+i N_{j}(\cdot) k_{j}\right\rangle\left\langle 1-i N_{l}(\cdot) k_{l}\right\rangle+O\left(k^{2}\right)= \\
1+\left\langle N_{j} N_{l}\right\rangle k_{j} k_{l}+O\left(k^{2}\right)=1+O\left(k^{2}\right)
\end{gathered}
$$

и нормировка скажется в разложении $\varphi_{0}(\cdot, k)$ лишь на членах порядка два и выше, которые на этом этапе не конкретизируются и отнесены в остаточный член.

\section{4. ВЫДЕЛЕНИЕ ГЛАВНОГО ЧЛЕНА АСИМПТОТИКИ}

4.1. Пусть $G(k, x, y, t)$ - функция Грина периодической задачи (2.5), т. е. ядро интегрального оператора $e^{-t A(k)}$. Через функцию Грина $G$ выражается фундаментальное решение $K(x, y, t)$, а именно,

$$
K(x, y, t)=\frac{1}{(2 \pi)^{d}} \int_{\square^{*}} e^{i k \cdot(x-y)} G(k, x, y, t) d k .
$$

Приведем вывод этой формулы, опираясь на блоховское представление операторной экспоненты $e^{-t A}$. В равенстве

$$
e^{-t A} f(x) \stackrel{(2.6)}{=} \frac{1}{(2 \pi)^{\frac{d}{2}}} \int_{\square^{*}} e^{i k \cdot x} e^{-t A(k)} \hat{f}(x, k) d k,
$$

используя определение функции Грина и преобразования $\hat{f}$, сделаем подстановки

$$
\begin{gathered}
e^{-t A(k)} \hat{f}(x, k)=\int_{\square} G(k, x, y, t) \hat{f}(y, k) d y, \\
\hat{f}(y, k)=\frac{1}{(2 \pi)^{\frac{d}{2}}} \sum_{h} f(y+h) e^{-i(y+h) \cdot k}
\end{gathered}
$$

и учтем периодичность $G(k, x, y, t)$ по $y$. Тогда

$$
\begin{gathered}
e^{-t A} f(x)=\frac{1}{(2 \pi)^{d}} \int_{\square^{*}} e^{i k \cdot x}\left(\sum_{h} \int_{\square} G(k, x, y+h, t) f(y+h) e^{-i(y+h) \cdot k} d y\right) d k= \\
=\frac{1}{(2 \pi)^{d}} \int_{\square^{*}} e^{i k \cdot x}\left(\int_{\mathbb{R}^{d}} G(k, x, y, t) f(y) e^{-i y \cdot k} d y\right) d k= \\
=\int_{\mathbb{R}^{d}}\left(\frac{1}{(2 \pi)^{d}} \int_{\square^{*}} e^{i k \cdot(x-y)} G(k, x, y, t) d k\right) f(y) d y=\int_{\mathbb{R}^{d}} K(x, y, t) f(y) d y,
\end{gathered}
$$

откуда следует представление (4.1) для ядра $K(x, y, t)$ интегрального оператора $e^{-t A}$. 
4.2. Отметим некоторые свойства собственных значений $\lambda_{j}(k)$ и собственных функций $\varphi_{j}(x, k)$ оператора $A(k)$.

Для собственных значений оператора сравнения $J(k)$ (см. (3.3)) выполнено неравенство

$$
\pi^{2}(2|n|-1)^{2} \leqslant(2 \pi n+k)^{2} \leqslant \pi^{2}(2|n|+1)^{2}, \quad k \in \square^{*}, \quad n \in \mathbb{Z}^{d} .
$$

Отсюда выводим оценку сверху для считающей функции этого оператора

$$
\sum_{(2 \pi n+k)^{2} \leqslant \lambda} 1 \leqslant c \lambda^{\frac{d}{2}}
$$

и аналогичная оценка верна для считающей функции оператора $A(k)$.

Важна также поточечная оценка для нормированных в $L^{2}(\square)$ собственных функций оператора $A(k)$

$$
\sup _{x \in \square}\left|\varphi_{j}(x, k)\right| \leqslant c\left(1+\lambda_{j}^{d}\right) .
$$

которая есть следствие предыдущей оценки. В самом деле, исходим из операторного неравенства

$$
A(k) \geqslant \nu J(k),
$$

которое влечет соответствующее неравенство для спектральных проекторов

$$
E_{A}(\lambda) \leqslant \nu^{-1} E_{J}(\lambda), \lambda>0 .
$$

Спектральный проектор $E_{A}(\lambda)$ - это интегральный оператор с ядром

$$
\sum_{\lambda_{j}(k) \leqslant \lambda} \varphi_{j}(k, x) \bar{\varphi}_{j}(k, y) .
$$

На диагонали (при $x=y$ ) выполняется поточечное неравенство

$$
\sum_{\lambda_{j}(k) \leqslant \lambda}\left|\varphi_{j}(k, x)\right|^{2} \leqslant \nu^{-1} \sum_{(2 \pi n+k)^{2} \leqslant \lambda} 1 \stackrel{(4.2)}{\leqslant} c \lambda^{\frac{d}{2}},
$$

из которого следует (4.3).

4.3. Оценка (4.3) позволяет представить функцию Грина в виде равномерно сходящегося ряда

$$
G(k, x, y, t)=\sum_{j=0}^{\infty} e^{-t \lambda_{j}(k)} \varphi_{j}(k, x) \bar{\varphi}_{j}(k, y) .
$$

Учитывая отмеченные в разделе 3 свойства собственных значений $\lambda_{j}(k)$, выделяем в этом разложении главное слагаемое, а остальные относим к остаточному члену. В результате получаем асимптотическое представление

$$
G(k, x, y, t)=e^{-t \lambda_{0}(k)} \varphi_{0}(k, x) \bar{\varphi}_{0}(k, y)+O\left(e^{-c_{0} t}\right), c_{0}>0,
$$

равномерное по $x, y \in \square, k \in \square^{*}$. Отсюда и из (4.1) следует асимптотическое представление

$$
K(x, y, t)=\frac{1}{(2 \pi)^{d}} \int_{\square^{*}} e^{i k \cdot(x-y)} e^{-t \lambda_{0}(k)} \varphi_{0}(x, k) \bar{\varphi}_{0}(y, k) d k+O\left(e^{-c_{0} t}\right),
$$

также равномерное по $x, y \in \mathbb{R}^{d}$. Здесь интеграл по всей ячейке $\square^{*}$ можно заменить на интеграл по достаточно малой окрестности нуля, где имеют место разложения (3.12). Интеграл по остальной части в силу леммы 3.2, (ii) можно отнести в остаточный член экспоненциального характера.

Итак, рассматривая в (4.4) интегрирование только по малой окрестности нуля, заменим $\varphi_{0}$ и $\lambda_{0}(k)$ приближенными выражениями $1+i N_{j} k_{j}, a^{0} k \cdot k$ и оценим соответствующую ошибку. Поскольку

$$
\int_{|k| \leqslant r_{0}} e^{-t \lambda_{0}(k)}|k|^{2} d k \leqslant \int_{\mathbb{R}^{d}} e^{-t \nu k^{2}}|k|^{2} d k=c t^{-\frac{d+2}{2}},
$$


то замена $\varphi_{0}$ на $1+i k_{j} N_{j}$ дает ошибку порядка $t^{-\frac{d+2}{2}}$. Остается оценить величину

$$
\int_{|k| \leqslant r_{0}}\left|e^{-t \lambda_{0}(k)}-e^{t a^{0} k \cdot k}\right| d k
$$

Учитывая неравенства $\lambda_{0}(k) \geqslant \nu k^{2}$ (см. (3.4)), $a^{0} k \cdot k \geqslant \nu k^{2}$ (см. (1.13)), выводим оценку

$$
\left|e^{-t \lambda(k)}-e^{-t a^{0} k \cdot k}\right| \leqslant t\left|\lambda_{0}(k)-a^{0} k \cdot k\right| e^{-t \nu k^{2}} \leqslant c_{1} t k^{4} e^{-t \nu k^{2}} .
$$

Поэтому величина (4.5) имеет порядок $t^{-\frac{(d+2)}{2}}$.

В результате из (4.4) получили представление

$$
\begin{gathered}
(2 \pi)^{d} K(x, y, t)=\int_{|k| \leqslant r_{0}} e^{i k \cdot(x-y)} e^{-t a^{0} k \cdot k}\left(1+i k_{j}\left(N_{j}(x)-N_{j}(y)\right)\right) d k+O\left(t^{-\frac{d+2}{2}}\right)= \\
\int_{\mathbb{R}^{d}} e^{i k \cdot(x-y)} e^{-t a^{0} k \cdot k}\left(1+i k_{j}\left(N_{j}(x)-N_{j}(y)\right)\right) d k+O\left(t^{-\frac{d+2}{2}}\right),
\end{gathered}
$$

где расширение области интегрирования приводит к изменению лишь остаточного члена. Последний интеграл легко вычисляется с помощью преобразования Фурье, что дает

$$
K(x, y, t)=K_{0}(x, y, t)+\left(N_{j}(x)-N_{j}(y)\right) \frac{\partial}{\partial x_{j}} K_{0}(x, y, t)+O\left(t^{-\frac{d+2}{2}}\right) .
$$

Отсюда огрублением следует поточечная оценка (1.7), так как

$$
\left(N_{j}(x)-N_{j}(y)\right) \frac{\partial}{\partial x_{j}} K_{0}(x, y, t)=\frac{N_{j}(x)-N_{j}(y)}{\sqrt{t}} \frac{b_{i j}\left(x_{i}-y_{i}\right)}{\sqrt{t}} K_{0}(x, y, t), \quad b=\frac{1}{4}\left(a^{0}\right)^{-1}
$$

(см. (1.8)), и в силу ограниченности $N_{j}$ имеем

$$
\left|\left(N_{j}(x)-N_{j}(y)\right) \frac{\partial}{\partial x_{j}} K_{0}\right| \leqslant c t^{-\frac{d+1}{2}} .
$$

Вместе с тем для приближения

$$
K_{1}(x, y, t)=K_{0}(x, y, t)+\left(N_{j}(x)-N_{j}(y)\right) \frac{\partial}{\partial x_{j}} K_{0}(x, y, t)
$$

доказана оценка погрешности с большей точностью, а именно,

$$
\left|K(x, y, t)-K_{1}(x, y, t)\right| \leqslant c t^{-\frac{d+2}{2}}, \quad x, y \in \mathbb{R}^{d}, \quad c=\operatorname{const}(d, \nu),
$$

объявленная раннее в (1.15).

4.4. Займемся выводом интегральных оценок. Будем использовать две оценки

$$
\begin{gathered}
\left|K-K_{1}\right| \leqslant c t^{-\frac{d+2}{2}}, \\
\left|K-K_{1}\right| \leqslant c t^{-\frac{d}{2}} e^{-c_{0} \frac{(x-y)^{2}}{t}} .
\end{gathered}
$$

Первая только что доказана, вторая следует непосредственно из конструкции приближения $K_{1}$ (см. (1.14)) и известной оценки Нэша-Аронсона

$$
0 \leqslant K(x, y, t) \leqslant c_{1} t^{-\frac{d}{2}} e^{-\frac{c_{0}(x-y)^{2}}{t}},
$$

где константы $c_{1}, c_{0}>0$ зависят только от размерности $d$ и константы эллиптичности $\nu$. Оценка (4.8) доказана, например, в [9, приложение к гл. II].

Для $\alpha=\frac{1}{2}+\delta, \delta>0$, имеем

$$
\int_{\mathbb{R}^{d}}\left|K-K_{1}\right| d y=\int_{|x-y| \leqslant t^{\alpha}}\left|K-K_{1}\right| d y+\int_{|x-y| \geqslant t^{\alpha}}\left|K-K_{1}\right| d y \leqslant
$$




$$
\leqslant \frac{c t^{\alpha d}}{t^{\frac{d+2}{2}}}+\int_{|z| \geqslant t^{\delta}} e^{-\lambda_{0} z^{2}} d z \leqslant \frac{1}{t^{1-\delta d}}+c e^{-\frac{\lambda_{0}}{2} t^{\delta}} \leqslant \frac{C}{t^{1-d \delta}}
$$

Взяв здесь $\delta=\frac{1}{2 d}$, получим

$$
\int_{\mathbb{R}^{d}}\left|K-K_{1}\right| d y \leqslant \frac{c}{\sqrt{t}} .
$$

Теперь вспомним, что функции $N_{j}$ ограничены и поэтому

$$
\int_{\mathbb{R}^{d}}\left|K_{0}-K_{1}\right| d y \leqslant c_{1} \int_{\mathbb{R}^{d}}\left|\nabla_{x} K_{0}\right| d y \leqslant \frac{c}{\sqrt{t}},
$$

что вместе с (4.10) приводит к оценке (1.9).

Выбирая достаточно малым $\delta$, из (4.9) можно получить и более точную, чем (4.10), оценку, а именно,

$$
\int_{\mathbb{R}^{d}}\left|K(x, y, t)-K_{1}(x, y, t)\right| d y \leqslant \frac{c}{t^{1-\sigma}},
$$

где $\sigma>0$ сколь угодно мало, $c=\operatorname{const}(d, \nu, \sigma)$.

4.5. Подводя итоги, сформулируем точно, что доказано для приближений $K_{0}(x, y, t), K_{1}(x, y, t)$ фундаментального решения уравнения диффузии (1.1). Сами приближения определены в (1.8) и $(1.14)$.

Теорема 4.1. В предположениях пункта 1.1 на матрииу а (у) справедливы поточечные оценки (1.7) и (1.15), а также интегральные оценки (1.9) и (4.11).

Как следствие из теоремы 4.1 по лемме 1.1 вытекает

Теорема 4.2. В предположениях и обозначениях раздела 1 имеют место следующие оценки приближений для операторной экспоненты $e^{-t A}$ :

$$
\left\|e^{-t A}-e^{-t A_{0}}\right\|_{L^{p}\left(\mathbb{R}^{d}\right) \rightarrow L^{p}\left(\mathbb{R}^{d}\right)} \leqslant \frac{c}{\sqrt{t}} \quad \forall p \in[1, \infty]
$$

c единой константой $c=\operatorname{const}(d, \nu)$,

$$
\left\|e^{-t A}-\left(e^{-t A_{0}}+N(\cdot) \cdot \nabla e^{-t A_{0}}-e^{-t A_{0}} \nabla^{*} \cdot N(\cdot)\right)\right\|_{L^{p}\left(\mathbb{R}^{d}\right) \rightarrow L^{p}\left(\mathbb{R}^{d}\right)} \leqslant \frac{c}{t^{1-\sigma}} \quad \forall p \in[1, \infty]
$$

с единой константой $c=\operatorname{const}(d, \nu, \sigma)$, где $\sigma>0$ сколь угодно мало, $\nabla-$ градиент по пространственным переменным, $\nabla^{*}=\operatorname{div}$.

При $p=2$, используя разные версии спектрального подхода, в $[5,15,21]$ доказано, что оценка (4.12) верна и без потери в показателе степени $t$, т. е. с $\sigma=0$.

Вернемся к оценке (1.16). Для ее получения нужно определить приближение $K_{2}$ следующего порядка и для разности $\left|K-K_{2}\right|$ доказать оценку типа (4.9), в которой справа стоит $\frac{c}{t^{2-d \delta}}$, а затем, огрубляя, вернуться к $\left|K-K_{1}\right|$. Чтобы определить $K_{2}$, необходимо брать более точное, чем в (3.12), тейлоровское разложение для собственного значения $\lambda_{0}(k)$ и собственной функции $\varphi_{0}(x, k)$. Кроме того, потребуется дополнительное условие на регулярность матрицы $a(y)$.

\section{5. ВСПОМОГАТЕЛЬНЫЕ ЗАДАЧИ НА ЯЧЕЙКЕ}

Чтобы найти приближения высокого порядка для фундаментального решения, необходимо рассмотреть наряду с (1.11) другие задачи на ячейке.

Введем индуктивно следующие вспомогательные объекты:

- последовательность 1-периодических функций $\left\{N_{\alpha}\right\}_{\alpha}$,

- последовательность констант $\left\{a_{\alpha}^{0}\right\}_{\alpha}$. 
Здесь $\alpha$ - мультииндекс длины $|\alpha|=m(m \in \mathbb{N})$ следующего вида:

$$
\alpha=\left(\alpha_{1}, \ldots, \alpha_{m}\right), \alpha_{1} \leqslant \alpha_{2} \leqslant \cdots \leqslant \alpha_{m}, \alpha_{j} \in\{1, \ldots, d\} \quad \forall j=\overline{1, m} .
$$

Договоримся о некоторых обозначениях, связанных с мультииндексами.

Индекс $\alpha_{\bar{s}}$ получается из $\alpha$ вычеркиванием компоненты $\alpha_{s}$, индекс $\alpha_{\overline{s p}}-$ вычеркиванием из $\alpha_{\bar{s}}$ компоненты $\alpha_{p}$ и т. д. Ясно, что $\left|\alpha_{\bar{s}}\right|=m-1,\left|\alpha_{\overline{s p}}\right|=m-2$ и т. д. Если индекс $\beta$ составлен из некоторых компонент индекса $\alpha$, то пишем $\beta \subset \alpha$, а индекс $\alpha_{\bar{\beta}}$ получается из $\alpha$ вычеркиванием компонент, входящих в $\beta$.

5.1. Ранее были определены функции $N_{\alpha}$ для индекса единичной длины. Это - решения $N_{j}$ задачи (1.11) с базисными векторами $e_{j}, j=1, \ldots, d$. Используя $N_{j}$ и $e_{j}$, можно задать усредненную матрицу $a^{0}$ из (1.10) следующими соотношениями:

$$
a^{0} e_{j}=\left\langle a\left(e_{j}+\nabla N_{j}\right)\right\rangle, \quad j=1, \ldots, d .
$$

Теперь определим функцию $N_{\alpha}$ для произвольного индекса $\alpha=\left(\alpha_{1}, \alpha_{2}\right)$ длины 2 как решение задачи

$$
\begin{gathered}
N_{\alpha} \in H_{\mathrm{per}}^{1}(\square), \quad\left\langle N_{\alpha}\right\rangle=0, \\
-\operatorname{div}\left(a \nabla N_{\alpha}\right)+\operatorname{div} \sum_{\alpha_{j} \subset \alpha} a e_{\alpha_{j}} N_{\alpha_{\bar{j}}}+\sum_{\alpha_{j} \subset \alpha} a e_{\alpha_{j}}\left(\nabla N_{\alpha_{\bar{j}}}+e_{\alpha_{\bar{j}}}\right)=\sum_{\alpha_{j} \subset \alpha} a^{0} e_{\alpha_{j}} e_{\alpha_{\bar{j}}} .
\end{gathered}
$$

Здесь $e_{\alpha_{j}}$ - базисный вектор и $N_{\alpha_{j}}$ - решение соответствующей задачи $(1.11) ; a^{0}$ - усредненная матрица; в сумме $\sum_{\alpha_{j} \subset \alpha}$ перебираются все попарно различные компоненты $\alpha_{j}$ мультииндекса $\alpha$.

В отличие от (1.11), для задачи (5.3) в общих предположениях на матрицу $a(y)$ нет $L^{\infty}$ ограниченности решения $N_{\alpha}$. Однако, это свойство можно обеспечить, если матрица $a(y)$ достаточна регулярна.

Задача (5.3) того же типа, что (1.12), и условие ее разрешимости имеет вид

$$
\left\langle\sum_{\alpha_{j} \subset \alpha} a e_{\alpha_{j}}\left(\nabla N_{\alpha_{\bar{j}}}+e_{\alpha_{\bar{j}}}\right)\right\rangle=\sum_{\alpha_{j} \subset \alpha} a^{0} e_{\alpha_{j}} e_{\alpha_{\bar{j}}} .
$$

Это условие выполнено в силу симметричности матриц $a, a^{0}$ и вытекающих из (5.2) соотношений

$$
\left\langle a\left(\nabla N_{\alpha_{\bar{j}}}+e_{\alpha_{\bar{j}}}\right)\right\rangle=a^{0} e_{\alpha_{\bar{j}}}, \quad \alpha_{j} \subset \alpha,
$$

для любого индекса $\alpha,|\alpha|=2$.

Для дальнейшего удобно ввести константы $a_{\alpha}^{0},|\alpha|=2, \alpha=\left(\alpha_{1}, \alpha_{2}\right)$, которые выражаются через коэффициенты матрицы $a^{0}$ следующим образом:

$$
a_{\alpha}^{0}=\left\{\begin{array}{lll}
2 a^{0} e_{\alpha_{1}} e_{\alpha_{2}}, & \text { если } & \alpha_{1} \neq \alpha_{2}, \\
a^{0} e_{\alpha_{1}} e_{\alpha_{1}}, & \text { если } & \alpha_{1}=\alpha_{2} .
\end{array}\right.
$$

Тогда правую часть (5.3) заменяет константа $a_{\alpha}^{0}$.

5.2. Функции $N_{\alpha}$ и константы $a_{\alpha}^{0}$ с индексом $\alpha,|\alpha|>2$, вводятся индуктивно по длине индекса $|\alpha|$.

Предположим, что определены все функции $N_{\beta},|\beta|<m$, а также все константы $\alpha_{\beta}^{0}$ с мультииндексом $\beta$ четной длины $|\beta|<m$. Тогда функция $N_{\alpha}$, где $\alpha$-любой индекс вида (5.1) длины $m$, есть решение одной из двух следующих задач в зависимости от того, четно или нечетно $\mathrm{m}$.

Если $|\alpha|=m=2 l+1, l=1,2, \ldots$, то

$$
\begin{gathered}
N_{\alpha} \in H_{\mathrm{per}}^{1}(\square),\left\langle N_{\alpha}\right\rangle=0, \\
-\operatorname{div} a \nabla N_{\alpha}-\operatorname{div} \sum_{\alpha_{j} \subset \alpha} a e_{\alpha_{j}} N_{\alpha_{\bar{j}}}-\sum_{\alpha_{j} \subset \alpha} a e_{\alpha_{j}}\left(\nabla N_{\alpha_{\bar{j}}}-\sum_{\alpha_{s} \subset \alpha_{\bar{j}}} e_{\alpha_{s}} N_{\alpha_{\bar{j} \bar{s}}}\right)=\sum_{|\beta|=2 p<m} a_{\beta}^{0} N_{\alpha_{\bar{\beta}}} .
\end{gathered}
$$

Если $|\alpha|=m=2 l, l=2,3, \ldots$, то

$$
N_{\alpha} \in H_{\text {per }}^{1}(\square),\left\langle N_{\alpha}\right\rangle=0,
$$

$-\operatorname{div} a \nabla N_{\alpha}+\operatorname{div} \sum_{\alpha_{j} \subset \alpha} a e_{\alpha_{j}} N_{\alpha_{\bar{j}}}+\sum_{\alpha_{j} \subset \alpha} a e_{\alpha_{j}}\left(\nabla N_{\alpha_{\bar{j}}}+\sum_{\alpha_{s} \subset \alpha_{\bar{j}}} e_{\alpha_{s}} N_{\alpha_{\bar{j} \bar{s}}}\right)=\sum_{|\beta|=2 p \leqslant m} a_{\beta}^{0} N_{\alpha_{\bar{\beta}}}$. 
В уравнении (5.5) появилась новая константа $a_{\alpha}^{0}$ с индексом $\alpha$ длины $|\alpha|=m=2 l$.

Дадим по поводу этих задач некоторые комментарии.

1. Суммирование в правых частях (5.4) и (5.5) идет по всем допустимым индексам $\beta \subset \alpha$ четной длины. По соглашению $N_{\gamma} \equiv 1$, если $|\gamma|=0$, т. е. $\gamma$ - пустой индекс. В суммах вида $\sum_{\gamma_{j} \subset \gamma}$ перебираются все попарно различные компоненты $\gamma_{j}$ мультииндекса $\gamma$.

2. Константа $a_{\alpha}^{0},|\alpha|=m$, выбрана в правой части (5.5) так, чтобы выполнялось условие разрешимости этой задачи, а именно

$$
\left\langle\sum_{\alpha_{j} \subset \alpha} a e_{\alpha_{j}}\left(\nabla N_{\alpha_{\bar{j}}}+\sum_{\alpha_{s} \subset \alpha_{\bar{j}}} e_{\alpha_{s}} N_{\alpha_{\bar{j} \bar{s}}}\right)\right\rangle=a_{\alpha}^{0}, \quad|\alpha|=m=2 l .
$$

Для формирования условия разрешимости (5.6) важно, что функции $N_{\alpha_{\bar{\beta}}}$, найденные на предыдущих этапах, имеют среднее $\left\langle N_{\alpha_{\bar{\beta}}}\right\rangle=0$.

Итак, задачи (5.5) для индексов $\alpha$ четной длины $|\alpha|=m$ разрешимы за счет согласованного выбора констант $a_{\alpha}^{0},|\alpha|=m$. Что касается разрешимости задач (5.4) для индексов $\alpha$ нечетной длины $|\alpha|=m$, то она обеспечена автоматически самим процессом введения на предшествующих этапах функций $\left\{N_{\alpha}\right\}$ и констант $\left\{a_{\alpha}^{0}\right\}$ с мультииндексами меньшей длины. Этот отнюдь не очевидный факт доказан в [21].

3. При условии, что матрица $a(y)$ гладкая, все решения задач (5.4)-(5.5) тоже гладкие и, в частности, ограничены.

\section{6. ПОЛНОЕ АНАЛИТИЧЕСКОЕ РАЗЛОЖЕНИЕ}

Конкретизируем аналитические разложения по $k$ для собственного значения $\lambda_{0}(k)$ и собственной функции $\varphi_{0}(x, k)$, о которых шла речь раньше в разделе 3.

Лемма 6.1. Найдется такое $\delta_{0}>0$, что для $|k|<\delta_{0}$ имеют место утверждения:

(i) собственное значение $\lambda_{0}(k)$ простое и является аналитической функцией, допускающей разложение

$$
\lambda_{0}(k)=a^{0} k \cdot k+\sum_{|\alpha|=2 p \geqslant 4} a_{\alpha}^{0} k^{\alpha}
$$

где матрица а о определена в (1.10), а константь $a_{\alpha}^{0}-$ в (5.6);

(ii) собственную функцию $\varphi_{0}(x, k)$ можно выбрать аналитической по $k$, так что верно разложение

$$
\varphi_{0}(x, k)=1+i k \cdot N(x)+\sum_{|\alpha|=2 p \geqslant 2} k^{\alpha} N_{\alpha}(x)+i \sum_{|\alpha|=2 p+1 \geqslant 3} k^{\alpha} N_{\alpha}(x),
$$

где $N=\left(N_{1}, \ldots, N_{d}\right)$-решение задачи (1.11), $N_{\alpha}(x),|\alpha|=2$, - решение задачи (5.3), $N_{\alpha}(x),|\alpha|=m \geqslant 3,-$ решение задачи (5.4) или (5.5) в зависимости от того, нечетно или четно число $\mathrm{m}$.

В разложениях (6.1) или (6.2) степень $k^{\alpha}$ для вектора $k=\left(k_{1}, \ldots, k_{d}\right)$ с мультииндексом $\alpha=$ $\left(\alpha_{1}, \ldots, \alpha_{m}\right)$ определяется как

а свойства мультииндекса $\alpha$ даны в (5.1).

$$
k^{\alpha}=k_{\alpha_{1}} k_{\alpha_{2}} \cdots k_{\alpha_{m}}
$$

Располагая разложениями (6.1) или (6.2), можно через их коэффициенты выразить величину $\nu(k)$ из (3.13). Поскольку

$$
\overline{\varphi_{0}(x, k)}=1+\sum_{|\alpha|=2 p \geqslant 2} k^{\alpha} N_{\alpha}(x)-i\left(k \cdot N(x)+\sum_{|\alpha|=2 p+1 \geqslant 3} k^{\alpha} N_{\alpha}(x)\right)
$$

и

$$
\nu(k)=\left\langle\varphi_{0}(\cdot, k) \overline{\varphi_{0}(\cdot, k)}\right\rangle=\int_{\square}\left[\left(1+\sum_{|\alpha|=2 p \geqslant 2}^{\infty} k^{\alpha} N_{\alpha}(x)\right)^{2}+\left(\sum_{|\alpha|=2 p+1 \geqslant 1}^{\infty} k^{\alpha} N_{\alpha}(x)\right)^{2}\right] d x,
$$


имеем в первом приближении $\nu(k)=1+O\left(k^{2}\right)$ или точно

$$
\begin{gathered}
\nu(k)=1+\sum_{|\alpha|=2 p \geqslant 2}^{\infty} k^{\alpha} \sum_{\beta \subset \alpha}\left\langle N_{\beta} N_{\alpha_{\bar{\beta}}}\right\rangle= \\
=1+\sum_{|\alpha|=2 p \geqslant 2}^{\infty} c_{\alpha} k^{\alpha},
\end{gathered}
$$

где

$$
c_{\alpha}=\sum_{\beta \subset \alpha, 1 \leqslant|\beta| \leqslant 2 p-1}\left\langle N_{\beta} N_{\alpha_{\bar{\beta}}}\right\rangle, \quad|\alpha|=2 p
$$

(при суммировании перебираются все допустимые попарно различные индексы $\beta \subset \alpha$ ).

Доказательство леммы 6.1. Остановимся коротко лишь на идентификации коэффициентов в указанных разложениях.

Благодаря свойству четности (3.5) аналитическая функция $\lambda_{0}(k)$ раскладывается в ряд только по четным степеням, что отражено в (6.1), а относительно разложения собственной функции можно утверждать

$$
\varphi_{0}(x, k)=1+i \sum_{|\alpha|=2 p+1 \geqslant 1} N_{\alpha}(x) k^{\alpha}+\sum_{|\alpha|=2 p \geqslant 2} N_{\alpha}(x) k^{\alpha},
$$

где все $N_{\alpha}(x)$ вещественнозначны. Ранее в разделе 3 мы уже отождествили матрицу $a^{0}$ из $(6.1)$ с усредненной матрицей, а коэффициенты $N_{\alpha}(x),|\alpha|=1$, из (6.5) - с решениями задачи (1.11). Действуем по той же схеме и далее.

Используя представление (2.4) для оператора $A(k)$, а также полученные на этот момент предварительные разложения (6.1) и (6.5), запишем равенство

$$
0=A(k) \varphi_{0}(x, k)-\lambda_{0}(k) \varphi_{0}(x, k),
$$

группируя в нем слагаемые с одной и той же степенью $k^{\alpha}$. Имеем

$$
\begin{gathered}
0=A(k) \varphi_{0}(x, k)-\lambda_{0}(k) \varphi_{0}(x, k)= \\
\sum_{j=1}^{n} i k_{j}\left[-\operatorname{div} a\left(\nabla N_{j}+e_{j}\right)\right]+ \\
+\sum_{|\alpha|=2} k^{\alpha}\left[-\operatorname{div} a \nabla N_{\alpha}+\operatorname{div} \sum_{\alpha_{j} \subset \alpha} a e_{\alpha_{j}} N_{\alpha_{\bar{j}}}+\sum_{\alpha_{j} \subset \alpha} a e_{\alpha_{j}} \cdot\left(\nabla N_{\alpha_{\bar{j}}}+e_{\alpha_{\bar{j}}}\right)\right. \\
\left.-\sum_{\alpha_{j} \subset \alpha}^{a} a^{0} e_{\alpha_{j}} e_{\alpha_{\bar{j}}}\right]+ \\
+\sum_{l=1|\alpha|=m=2 l+1}^{\infty} i k^{\alpha}\left[-\operatorname{div} a \nabla N_{\alpha}-\operatorname{div} \sum_{\alpha_{j} \subset \alpha} a e_{\alpha_{j}} N_{\alpha_{\bar{j}}}-\right. \\
\left.-\sum_{\alpha_{j} \subset \alpha} a e_{\alpha_{j}} \cdot\left(\nabla N_{\alpha_{\bar{j}}}-\sum_{\alpha_{s} \subset \alpha_{\bar{j}}} e_{\alpha_{s}} N_{\alpha_{\bar{j} \bar{s}}}\right)-\sum_{|\beta|=2 p<m} a_{\beta}^{0} N_{\alpha_{\bar{\beta}}}\right]+ \\
+\sum_{l=2}^{\infty} \sum_{|\alpha|=m=2 l} k^{\alpha}\left[-\operatorname{div} a \nabla N_{\alpha}+\operatorname{div} \sum_{\alpha_{j} \subset \alpha} a e_{\alpha_{j}} N_{\alpha_{\bar{j}}}+\sum_{\alpha_{j} \subset \alpha} a e_{\alpha_{j}}\left(\nabla N_{\alpha_{\bar{j}}}+\right.\right. \\
\left.\left.+\sum_{\alpha_{s} \subset \alpha_{\bar{j}}} e_{\alpha_{s}} N_{\alpha_{\bar{j} \bar{s}}}\right)-\sum_{|\beta|=2 p \leqslant m} a_{\beta}^{0} N_{\alpha_{\bar{\beta}}}\right] .
\end{gathered}
$$

Здесь действует соглашение о работе с мультииндексами (см. начало пункта 5.1) и, как прежде (см. комментарии в конце пункта 5.1), в сумме $\sum_{\gamma_{j} \subset \gamma}$ перебираются все попарно различные компоненты $\gamma_{j}$ мультииндекса $\gamma$.

Приравнивая нулю коэффициенты при различных степенях $k^{\alpha}$ в (6.6), $|\alpha| \geqslant 1$, выводим искомые формулы для коэффициентов разложения $N_{\alpha}(x)$ и $a_{\alpha}^{0}$.

\section{7. ПРИБЛИЖЕНИЯ ВЫСОКОГО ПОРЯДКА}

Ранее были построены $K_{0}(x, y, t), K_{1}(x, y, t)$ - нулевое и первое приближения к фундаментальному решению $K(x, y, t)$ уравнения (1.1), так что выполнены оценки (1.7) и (1.15) порядка 
$O\left(t^{-\frac{d+1}{2}}\right)$ и $O\left(t^{-\frac{d+2}{2}}\right)$ соответственно. Согласно $(1.14) K_{1}(x, y, t)$ получается из $K_{0}(x, y, t)$ добавлением первого корректора

$$
\begin{gathered}
K_{1}(x, y, t)=K_{0}(x, y, t)+S_{1}(x, y, t), \\
S_{1}(x, y, t)=\left(N_{j}(x)-N_{j}(y)\right) \frac{\partial}{\partial x_{j}} K_{0}(x, y, t) .
\end{gathered}
$$

Аналогичным образом строятся приближения следующих порядков $O\left(t^{-\frac{d+3}{2}}\right)$ и т. д. Например, $K_{2}(x, y, t)$ получается из $K_{1}(x, y, t)$ добавлением второго корректора $S_{2}(x, y, t)$. Объясним, как находятся корректоры на каждом очередном шаге.

Если собственным значениям (3.1) соответствуют собственные функции (3.2), не образующие ортонормированный базис, то формула (4.4) требует поправки, если мы интересуемся приближениями, более точными, чем $K_{0}(x, y, t)$ и $K_{1}(x, y, t)$.

Введем базис $\left\{\varphi_{j}^{*}(x, k)\right\}_{j=0}^{\infty}$, сопряженный к базису (3.2), так что

$$
\left\langle\varphi_{j}^{*}(\cdot, k) \overline{\varphi_{m}(\cdot, k)}\right\rangle=\delta_{j m} .
$$

В частности,

$$
\varphi_{0}^{*}(x, k)=\nu(k)^{-1} \varphi_{0}(x, k), \quad \nu(k)=\left\langle\varphi_{0}(\cdot, k) \overline{\varphi_{0}(\cdot, k)}\right\rangle .
$$

Тогда

$$
e^{-t A(k)}=\sum_{j=0}^{\infty} e^{-t \lambda_{j}(k)} \varphi_{j}(x, k) \times \varphi_{j}^{*}(x, k),
$$

где для заданных функций $\psi_{1}, \psi_{2} \in L^{2}(\square)$ проектор $\psi_{1} \times \psi_{2}$ в $L^{2}(\square)$ действует по правилу

$$
\left(\psi_{1} \times \psi_{2}\right) v=\psi_{1} v \bar{\psi}_{2} d x .
$$

Для ядра экспоненты $e^{-t A(k)}$ так же, как в пункте 4.3, устанавливаем равенство

$$
G(k, x, y, t)=e^{-t \lambda_{0}(k)} \varphi_{0}(k, x) \bar{\varphi}_{0}(k, y) \nu(k)^{-1}+O\left(e^{-c_{0} t}\right), c_{0}>0,
$$

равномерное по $x, y \in \square, k \in \square^{*}$. Отсюда и из (4.1) следует представление

$$
K(x, y, t)=\frac{1}{(2 \pi)^{d}} \int_{\square^{*}} e^{i k \cdot(x-y)} e^{-t \lambda_{0}(k)} \varphi_{0}(x, k) \bar{\varphi}_{0}(y, k) \nu(k)^{-1} d k+O\left(e^{-c_{0} t}\right),
$$

где функция $\varphi_{0}(x, k)$ описана в лемме 6.1. Последний интеграл по ячейке $\square *$ можно заменить на интеграл по достаточно малой окрестности нуля, в которой имеют место разложения (6.1), (6.2), (6.3). Возьмем эти разложения с остаточным членом шестого, третьего и четвертого порядков соответственно, а именно,

$$
\begin{gathered}
\lambda_{0}(k)=a^{0} k \cdot k+\sum_{|\alpha|=4} a_{\alpha}^{0} k^{\alpha}+O\left(k^{6}\right), \quad \varphi_{0}(x, k)=1+i N(x) \cdot k+\sum_{|\alpha|=2} k^{\alpha} N_{\alpha}(x)+O\left(k^{3}\right), \\
\nu(k)=1+\sum_{|\alpha|=2} c_{\alpha} k^{\alpha}+O\left(k^{4}\right),
\end{gathered}
$$

а также выделим в первом разложении главный член

$$
\lambda_{0}(k)=a^{0} k \cdot k+\mu(k), \quad \mu(k)=\sum_{|\alpha|=4} a_{\alpha}^{0} k^{\alpha}+O\left(k^{6}\right),
$$

и запишем следующие разложения:

$$
\begin{gathered}
\varphi_{0}(x, k) \bar{\varphi}_{0}(y, k) \nu(k)^{-1}=1+i k \cdot(N(x)-N(y))+k_{j} k_{l} N_{j}(x) N_{l}(y)+ \\
\sum_{|\alpha|=2} k^{\alpha}\left(N_{\alpha}(x)+N_{\alpha}(y)-c_{\alpha}\right)+O\left(k^{3}\right) \equiv s_{2}(x, y, k)+O\left(k^{3}\right), \\
e^{-t \mu(k)}=1-t \sum_{|\alpha|=4} a_{\alpha}^{0} k^{\alpha}+t O\left(k^{6}\right) .
\end{gathered}
$$


Тогда аналогом (4.6) будет равенство

$$
\begin{gathered}
(2 \pi)^{d} K(x, y, t)=\int_{|k| \leqslant r_{0}} e^{i k \cdot(x-y)} e^{-t a^{0} k \cdot k} e^{-t \mu(k)} s_{2}(x, y, k) d k+O\left(t^{-\frac{d+3}{2}}\right)= \\
\int_{\mathbb{R}^{d}} e^{i k \cdot(x-y)} e^{-t a^{0} k \cdot k}\left(s_{2}(x, y, k)-t \sum_{|\alpha|=4} a_{\alpha}^{0} k^{\alpha}\right) d k+O\left(t^{-\frac{d+3}{2}}\right),
\end{gathered}
$$

так как

$$
\begin{gathered}
\int_{|k| \leqslant r_{0}} e^{-t \lambda_{0}(k)}|k|^{3} d k \leqslant \int_{\mathbb{R}^{d}} e^{-t \nu k^{2}}|k|^{3} d k=c t^{-\frac{d+3}{2}}, \\
\int_{|k| \leqslant r_{0}} e^{-t \lambda_{0}(k)} t|k|^{6} d k \leqslant \int_{\mathbb{R}^{d}} t|k|^{2} e^{-t \nu k^{2}}|k|^{4} d k=\tilde{c} t^{-\frac{d+4}{2}} .
\end{gathered}
$$

Последний интеграл в (7.3) вычисляется (по известным формулам преобразования Фурье) с учетом выражения для $s_{2}(x, y, k)$ из $(7.2)$ и дает приближение $K_{2}(x, y, t)$. В итоге из (7.3) следуют равенства

$$
\begin{gathered}
K(x, y, t)=K_{2}(x, y, t)+O\left(t^{-\frac{d+3}{2}}\right), \\
K_{2}(x, y, t)=K_{1}(x, y, t)+S_{2}(x, y, t), \\
S_{2}(x, y, t)=-N_{j}(x) N_{j}(y) \frac{\partial^{2}}{\partial x_{j} \partial x_{l}} K_{0}(x, y, t)- \\
\sum_{|\alpha|=2}\left(N_{\alpha}(x)+N_{\alpha}(y)-c_{\alpha}\right) D_{x}^{\alpha} K_{0}(x, y, t)-t \sum_{|\alpha|=4} a_{\alpha}^{0} D_{x}^{\alpha} K_{0}(x, y, t) .
\end{gathered}
$$

Здесь и всюду далее для мультииндекса $\alpha=\left(\alpha_{1}, \ldots, \alpha_{m}\right)$ (см. (5.1))

есть соответствующая мультипроизводная.

$$
D_{x}^{\alpha}=\frac{\partial}{\partial x_{\alpha_{1}}} \cdots \frac{\partial}{\partial x_{\alpha_{m}}}
$$

Если в (7.1) взять одно разложение более точным, а именно,

$$
\varphi_{0}(x, k)=1+i N(x) \cdot k+\sum_{|\alpha|=2} k^{\alpha} N_{\alpha}(x)+i \sum_{|\alpha|=3} k^{\alpha} N_{\alpha}(x)+O\left(k^{4}\right),
$$

то аналогичные вычисления приведут к формулам

$$
\begin{gathered}
K(x, y, t)=K_{3}(x, y, t)+O\left(t^{-\frac{d+4}{2}}\right), \\
K_{3}(x, y, t)=K_{2}(x, y, t)+S_{3}(x, y, t), \\
S_{3}(x, y, t)=\sum_{|\alpha|=2}\left(-N_{j}(x) N_{\alpha}(y)+N_{j}(y) N_{\alpha}(x)+c_{\alpha} N_{j}(x)-c_{\alpha} N_{j}(y)\right) \frac{\partial}{\partial x_{j}} D_{x}^{\alpha} K_{0}(x, y, t)- \\
\sum_{|\alpha|=3}\left(N_{\alpha}(x)-N_{\alpha}(y)\right) D_{x}^{\alpha} K_{0}(x, y, t) .
\end{gathered}
$$

Таким образом, записывая разложения типа (7.1) с остаточными членами подходящего порядка, получаем корректоры $S_{j}(x, y, t), j \geqslant 2$, которые формируют последовательно приближения $K_{j}(x, y, t)$. При этом справедливы оценки

$$
\left|K(x, y, t)-K_{j}(x, y, t)\right| \leqslant c_{j} t^{-\frac{d+j+1}{2}}, \quad j=2,3, \ldots
$$

Эти поточечные оценки будут равномерными по $x$ и $y$, если в проведенных выше формально выкладках все участвующие функции

$$
N_{\alpha}(x),|\alpha| \geqslant 2, \quad \text { ограничены. }
$$

Чтобы обеспечить свойство (7.9), достаточно предположить, что $a(x)=\left\{a_{j l}(x)\right\}-$ липшицева матрица, т. е.

$$
\left|a_{j l}(x)-a_{j l}\left(x^{\prime}\right)\right| \leqslant c_{L}\left|x-x^{\prime}\right|, \quad x, x^{\prime} \in \square, \quad 1 \leqslant j, l \leqslant d .
$$


Справедливо следующее вспомогательное утверждение, доказательство которого вынесено в раздел 9.

Лемма 7.1. Нз липшицевости матрицьь $a(x)$ следует, ито $N_{\alpha} \in L^{\infty}(\square),|\alpha| \geqslant 2$.

Фактически доказана

Теорема 7.1. При выполнении условия липшицевости (7.10) описанная выше процедура построения приближений $K_{j}(x, y, t), j=2,3, \ldots$, для фундаментального решения $K(x, y, t)$ уравнения (1.1) приводит к равномерным поточечным оценкам (7.8) с константами $c_{j}=$ const $\left(d, \nu, c_{L}\right)$. Приближения $K_{2}(x, y, t)$ и $K_{3}(x, y, t)$ заданы явно в (7.4), (7.5) и (7.6), (7.7) соответственно через компоненты разложений (6.1)-(6.3) и фундаментальное решение (1.8).

Из поточечных оценок (7.8) выводятся соответствующие интегральные оценки, как показано в разделе 4 . В соответствии с аппроксимациями фундаментального решения строятся аппроксимации операторной экспоненты $e^{-t A}$ с оценкой погрешности по операторной $L^{p}$-норме, $1 \leqslant p \leqslant \infty$. Как пример можно рассмотреть теорему 4.2.

Аппроксимации высокого порядка к операторной экспоненте по операторной $L^{2}$-норме с несколько разных сторон изучались ранее: порядка $O\left(t^{-\frac{3}{2}}\right)$ в [6] и порядка $O\left(t^{-\frac{n}{2}}\right), n=1,2, \ldots$, в [21].

Из оценок (7.8) следуют аналоги вероятностной оценки Берри-Эссена с высоким порядком приближения плотности распределения случайной величины $\frac{\xi^{t}-y}{\sqrt{t}}$, связанной с уравнением (1.1), см. пункт 1.4. и оценки (1.18), (1.19).

\section{8. УРАВНЕНИЯ С $\varepsilon$-ПЕРИОДИЧЕСКИМИ КОЭФФИЦИЕНТАМИ}

Рассмотрим задачу Коши для функции $u^{\varepsilon}=u^{\varepsilon}(x, t), x \in \mathbb{R}^{d}, t \geqslant 0$ :

$$
\left\{\begin{array}{l}
\frac{\partial u^{\varepsilon}}{\partial t}+A_{\varepsilon} u^{\varepsilon}=0, t>0, \\
\left.u^{\varepsilon}\right|_{t=0}=f
\end{array}\right.
$$

где $A_{\varepsilon}=-\operatorname{div}\left(a_{\varepsilon} \nabla\right), a_{\varepsilon}(x)=a\left(\frac{x}{\varepsilon}\right), a(y)-1$-периодическая измеримая симметрическая матрица, удовлетворяющая условию (1.2).

Автомодельная замена

$$
y=\frac{x}{\varepsilon}, \quad \tau=\frac{t}{\varepsilon^{2}}
$$

сводит задачу с $\varepsilon$-периодической матрицей $a\left(\frac{x}{\varepsilon}\right)$ к задаче с 1-периодической матрицей $a(y)$. Именно, функция

$$
z^{\varepsilon}(y, \tau)=u^{\varepsilon}\left(\varepsilon y, \varepsilon^{2} \tau\right)
$$

есть решение задачи Коши

$$
\left\{\begin{array}{l}
\frac{\partial z^{\varepsilon}}{\partial \tau}+A z^{\varepsilon}=0, \tau>0, \\
\left.z^{\varepsilon}\right|_{\tau=0}=f_{\varepsilon}, \quad f_{\varepsilon}(y)=f(\varepsilon y) .
\end{array}\right.
$$

Тогда $z^{\varepsilon}(y, \tau)=e^{-\tau A} f_{\varepsilon}$, или в обозначениях раздела 1

$$
z^{\varepsilon}(y, \tau)=\int_{\mathbb{R}^{d}} K\left(y, y^{\prime}, \tau\right) f\left(\varepsilon y^{\prime}\right) d y^{\prime} .
$$

Обозначим через $K_{\varepsilon}\left(x, x^{\prime}, t\right)$ фундаментальное решение для оператора $\frac{\partial}{\partial t}+A_{\varepsilon}$, т. е. ядро интегрального оператора $e^{-t A_{\varepsilon}}$. Тогда

$$
u^{\varepsilon}(x, t)=\int_{\mathbb{R}^{d}} K_{\varepsilon}\left(x, x^{\prime}, t\right) f\left(x^{\prime}\right) d x^{\prime} .
$$


С другой стороны,

$$
\begin{aligned}
u^{\varepsilon}(x, t)= & z^{\varepsilon}\left(\frac{x}{\varepsilon}, \frac{t}{\varepsilon^{2}}\right) \stackrel{(8.1)}{=} \int_{\mathbb{R}^{d}} K\left(\frac{x}{\varepsilon}, y^{\prime}, \frac{t}{\varepsilon^{2}}\right) f\left(\varepsilon y^{\prime}\right) d y^{\prime}= \\
& \int_{\mathbb{R}^{d}} K\left(\frac{x}{\varepsilon}, \frac{x^{\prime}}{\varepsilon}, \frac{t}{\varepsilon^{2}}\right) f\left(x^{\prime}\right) \varepsilon^{-d} d x^{\prime} .
\end{aligned}
$$

Отсюда следует равенство

$$
K_{\varepsilon}\left(x, x^{\prime}, t\right)=\varepsilon^{-d} K\left(\frac{x}{\varepsilon}, \frac{x^{\prime}}{\varepsilon}, \frac{t}{\varepsilon^{2}}\right) .
$$

Непосредственно из формулы (1.8) видно, что

$$
\varepsilon^{-d} K_{0}\left(\frac{x}{\varepsilon}, \frac{x^{\prime}}{\varepsilon}, \frac{t}{\varepsilon^{2}}\right)=K_{0}\left(x, x^{\prime}, t\right) .
$$

Поэтому из оценки (1.7) последовательно выводим

$$
\begin{gathered}
\left|K\left(\frac{x}{\varepsilon}, \frac{x^{\prime}}{\varepsilon}, \frac{t}{\varepsilon^{2}}\right)-K_{0}\left(\frac{x}{\varepsilon}, \frac{x^{\prime}}{\varepsilon}, \frac{t}{\varepsilon^{2}}\right)\right| \leqslant c \frac{\varepsilon^{d+1}}{t^{\frac{d+1}{2}}}, \\
\left|\varepsilon^{-d} K\left(\frac{x}{\varepsilon}, \frac{x^{\prime}}{\varepsilon}, \frac{t}{\varepsilon^{2}}\right)-\varepsilon^{-d} K_{0}\left(\frac{x}{\varepsilon}, \frac{x^{\prime}}{\varepsilon}, \frac{t}{\varepsilon^{2}}\right)\right| \leqslant \varepsilon \frac{c}{t^{\frac{d+1}{2}}},
\end{gathered}
$$

откуда, в силу (8.2) и (8.3),

$$
\left|K_{\varepsilon}\left(x, x^{\prime}, t\right)-K_{0}\left(x, x^{\prime}, t\right)\right| \leqslant \varepsilon \frac{c}{t^{\frac{d+1}{2}}},
$$

Аналогично из (1.9) вытекает интегральная оценка

$$
\int_{\mathbb{R}^{d}}\left|K_{\varepsilon}\left(x, x^{\prime}, t\right)-K_{0}\left(x, x^{\prime}, t\right)\right| d x^{\prime} \leqslant \varepsilon \frac{c}{\sqrt{t}},
$$

из которой по лемме 1.1 уже можно вывести операторную оценку погрешности усреднения

$$
\left\|e^{-t A_{\varepsilon}}-e^{-t A_{0}}\right\|_{L^{p}\left(\mathbb{R}^{d}\right) \rightarrow L^{p}\left(\mathbb{R}^{d}\right)} \leqslant \varepsilon \frac{c}{\sqrt{t}} \quad \forall p \in[1, \infty]
$$

с единой константой для всех $p$.

В качестве следствия укажем оценку

$$
\left\|\left(A_{\varepsilon}+1\right)^{-1}-\left(A_{0}+1\right)^{-1}\right\|_{L^{p}\left(\mathbb{R}^{d}\right) \rightarrow L^{p}\left(\mathbb{R}^{d}\right)} \leqslant c \varepsilon \quad \forall p \in[1, \infty]
$$

для разности резольвент исходного эллиптического оператора $A_{\varepsilon}$ и усредненного оператора $A_{0}$.

Чтобы получить (8.5) из (8.4), надо воспользоваться известным представлением резольвенты через полугруппу

$$
(A+1)^{-1}=\int_{0}^{\infty} e^{-t} e^{-t A} d t
$$

Действительно,

$$
\left\|\left(A_{\varepsilon}+1\right)^{-1}-\left(A_{0}+1\right)^{-1}\right\| \leqslant \int_{0}^{\infty} e^{-t}\left\|e^{-t A_{\varepsilon}}-e^{-t A_{0}}\right\| d t \leqslant 2 \varepsilon+C \varepsilon \int_{\varepsilon}^{\infty} \frac{e^{-t}}{\sqrt{t}} d t \leqslant c \varepsilon .
$$

При $p=2$ оценка (8.5) впервые была доказана в [4]. 


\section{9. ДОКАЗАТЕЛЬСТВО ЛЕМмЫ 7.1}

Свойство (7.9) доказывается индуктивно по длине мультииндекса $\alpha,|\alpha| \geqslant 2$. Достаточно подробно рассмотреть случай $|\alpha|=2$.

Сначала докажем свойство, касающееся решений задачи (1.11), а именно,

$$
\frac{\partial N_{j}}{\partial x_{l}} \in L^{\infty}(\square), \quad 1 \leqslant j, l \leqslant d,
$$

при условии, что матрица $a(x)$ липшицева. Для этого продифференцируем уравнение $(1.11)$ для фиксированного $j$, что возможно благодаря липшицевости матрицы $a(x)$ (напомним, что по теореме Радемахера для п.в. $x \in \square$ существует градиент $\left.\nabla a_{j l} \in L^{\infty}(\square)^{d}\right)$. Получим уравнения

$$
\operatorname{div}\left[a(x) \nabla \frac{\partial N_{j}(x)}{\partial x_{m}}+\frac{\partial a(x)}{\partial x_{m}}\left(e_{j}+\nabla N_{j}(x)\right)\right]=0, \quad m=1, \ldots d .
$$

Введем обозначение $v_{m}=\frac{\partial N_{j}}{\partial x_{m}}$ и перепишем эти уравнения как систему относительно вектора $v=\left\{v_{m}\right\}$,

$$
\operatorname{div}\left[a(x) \nabla v_{m}+\frac{\partial a(x)}{\partial x_{m}}\left(e_{j}+v\right)\right]=0, \quad m=1, \ldots d .
$$

Система относится к классу, для которого имеет место векторный аналог принципа максимума (см. [14, гл. VII]). Таким образом, свойство (9.1) установлено.

Теперь рассмотрим уравнение (5.3) для произвольного фиксированного $\alpha$. В соответствии со структурой его «правой части» и в силу того, что $a_{j l}, N_{j}, \frac{\partial N_{j}(x)}{\partial x_{m}} \in L^{\infty}(\square)$, к уравнению применим принцип максимума, что дает ограниченность решения $N_{\alpha}$ этого уравнения. Кроме того, благодаря липшицевости матрицы $a(x)$, те же рассуждения, что выше, приводят к заключению: $\nabla N_{\alpha} \in$ $L^{\infty}(\square)^{d}$.

Далее по индукции можно переходить к рассмотрению уравнения (5.4) для произвольного фиксированного $\alpha$ длины $|\alpha|=3$ и применять прежние аргументы. Лемма доказана.

Замечание. Ограниченность градиента решения задачи на ячейке (1.11) при условии, что матрица $a(x)$ липшицева, отмечалась и доказывалась ранее в [22].

\section{СПИСОК ЛИТЕРАТУРЫ}

1. Александрова И.А. Спектральный метод в асимптотических задачах диффузии со сносом// Мат. заметки. - 1996. - 59, № 5. - С. 768-770.

2. Беляев А. Ю. Волны сжатия в жидкости с пузырьками воздуха// Прикл. мат. мех. $-1988 .-52$, № $3 .-$ C. $444-449$.

3. Беляев А. Ю. Усреднение в задачах теории фильтрации. - М.: Наука, 2004.

4. Бирман М. С., Суслина Т. А. Периодические дифференциальные операторы второго порядка. Пороговые свойства и усреднения// Алгебра и анализ. - 2003. - 15, № 5. - С. 1-108.

5. Василевская E.C. Усреднение параболической задачи Коши с периодическими коэффициентами при учете корректора// Алгебра и анализ. - 2008. - 21, № 1. - С. 3-60.

6. Василевская E.C., Суслина T. A. Пороговые аппроксимации факторизованного самосопряженного операторного семейства с учетом первого и второго корректоров// Алгебра и анализ. -2011 . -23 , № 2. C. $102-146$.

7. Жиков В. В. Асимптотическое поведение и стабилизация решений параболического уравнения второго порядка с младшими членами// Тр. Моск. Мат. об-ва. - 1983. - 46. - С. 69-98.

8. Жиков В.В. Спектральный подход к асимптотическим задачам диффузии// Дифф. уравн. - 1989.25, № 1. - С. 44-50.

9. Жиков В.В., Козлов С. М., Олейник О.А. Усреднение дифференциальных операторов. - М.: Наука, 1993.

10. Жиков В.В., Пастухова С.Е. Об операторных оценках в теории усреднения// Усп. мат. наук.2016. - 71, № 3. - С. 27-122.

11. Като T. Теория возмущений линейных операторов. - М.: Мир, 1972.

12. Киндерлерер Д., Стампаккья Г. Введение в вариационные неравенства и их приложения. - М.: Мир, 1983.

13. Коротков В. Б. Интегральные операторы. - Новосибирск: Наука, 1983. 
14. Ладыженская О.А., Уральцеева Н.Н. Линейные и квазилинейные уравнения эллиптического типа. М.: Наука, 1973.

15. Пастухова C.E. Аппроксимации операторной экспоненты в периодической задаче диффузии со сносом// Мат. сб. - 2013. - 204, № 2. - С. 133-160.

16. Севостьянова E. B. Асимптотическое разложение решения эллиптического уравнения второго порядка с периодическими быстро осциллирующими коэффициентами// Мат. сб. - 1981. - 115, № 2. - С. 204222.

17. Суслина T. А. Об усреднении периодических параболических систем// Функц. анализ и его прилож. 2004. - 38, № 4. - С. 86-90.

18. Феллер В. Введение в теорию вероятностей и ее приложения. Т. 2. - М.: Мир, 1967.

19. Bensousan A., Lions J.L., Papanicolaou G. Asymptotic Analysis for Periodic Structure. - Amsterdam: North Holland, 1978.

20. Ortega J. H., Zuazua E. Large time behavior in $\mathbb{R}^{d}$ for linear parabolic equations with periodic coefficients// Asymptot. Anal. - 2000. - 22, № 1. - C. 51-85.

21. Pastukhova S.E. Approximations of the exponential of an operator with periodic coefficients// J. Math. Sci. (N.Y.). - 2012. - 181, № 5. - C. 668-700.

22. Pastukhova S. E., Tikhomirov R. N. Error estimates of homogenization in the Neumann boundary problem for an elliptic equation with multiscale coefficients// J. Math. Sci. (N.Y.). - 2016. - 216, № 2. - C. 325344.

23. Zhikov V.V., Pastukhova S.E. Estimates of homogenization for a parabolic equation with periodic coefficients// Russ. J. Math. Phys. - 2006. - 13, № 2. - C. 224-237.

24. Zhikov V. V., Pastukhova S. E. Bloch principle for elliptic differential operators with periodic coefficients// Russ. J. Math. Phys. - 2016. - 23, № 2. - C. 257-277.

\title{
Василий Васильевич Жиков
}

Владимирский государственный университет им. А. Г. и Н. Г. Столетовых

600000, г. Владимир, ул. Горького, 87

Светлана Евгеньевна Пастухова

Московский технологический университет (МИРЭА)

119454, Москва, просп. Вернадского, 78

E-mail: pas-se@yandex.ru

\section{Large Time Asymptotics of Fundamental Solution for the Diffusion Equation in Periodic Medium and Its Application to Estimates in the Theory of Averaging}

\author{
(c) 2017 V. V. Zhikov, S. E. Pastukhova
}

\begin{abstract}
The diffusion equation is considered in an infinite 1-periodic medium. For its fundamental solution we find approximations at large values of time $t$. Precision of approximations has pointwise and integral estimates of orders $O\left(t^{-\frac{d+j+1}{2}}\right)$ and $O\left(t^{-\frac{j+1}{2}}\right), j=0,1, \ldots$, respectively. Approximations are constructed based on the known fundamental solution of the averaged equation with constant coefficients, its derivatives, and solutions of a family of auxiliary problems on the periodicity cell. The family of problems on the cell is generated recurrently. These results are used for construction of approximations of the operator exponential of the diffusion equation with precision estimates in operator norms in $L^{p}$-spaces, $1 \leqslant p \leqslant \infty$. For the analogous equation in an $\varepsilon$-periodic medium (here $\varepsilon$ is a small parameter) we obtain approximations of the operator exponential in $L^{p}$-operator norms for a fixed time with precision of order $O\left(\varepsilon^{n}\right), n=1,2, \ldots$
\end{abstract}




\section{REFERENCES}

1. I. A. Aleksandrova, "Spektralnyi metod v asimptoticheskikh zadachakh diffuzii so snosom" [Spectral method in asymptotic problems of diffusion with drift], Mat. zametki [Math. Notes], 1996, 59, No. 5, 768-770 (in Russian).

2. A. Iu. Beliaev, "Volny szhatiia v zhidkosti s puzyrkami vozdukha" [Waves of compression in a fluid with air bubbles], Prikl. mat. mekh. [Appl. Math. Mech.], 1988, 52, No. 3, 444-449 (in Russian).

3. A. Iu. Beliaev, Usrednenie v zadachakh teorii filtratsii [Averaging in Problems of the Filtration Theory], Nauka, Moscow, 2004 (in Russian).

4. M.S. Birman and T. A. Suslina, "Periodicheskie differentsialnye operatory vtorogo poriadka. Porogovye svoistva i usredneniia" [Periodic differential operators of second order. Threshold properties and averaging], Algebra $i$ analiz [Algebra Anal.], 2003, 15, No. 5, 1-108 (in Russian).

5. E. S. Vasilevskaia, "Usrednenie parabolicheskoi zadachi Koshi s periodicheskimi koeffitsientami pri uchete korrektora" [Averaging of parabolic Cauchy problem with periodic coefficients using a corrector], Algebra $i$ analiz [Algebra Anal.], 2008, 21, No. 1, 3-60 (in Russian).

6. E.S. Vasilevskaia and T.A. Suslina, "Porogovye approksimatsii faktorizovannogo samosopriazhennogo operatornogo semeistva s uchetom pervogo i vtorogo korrektorov" [Threshold approximations of a factorized self-adjoint operator family using the first and the second correctors], Algebra $i$ analiz [Algebra Anal.], 2011, 23, No. 2, 102-146 (in Russian).

7. V.V. Zhikov, "Asimptoticheskoe povedenie i stabilizatsiia reshenii parabolicheskogo uravneniia vtorogo poriadka s mladshimi chlenami" [Asymptotic behavior and stabilization of solutions of a second-order parabolic equation with lower-order terms], Tr. Mosk. Mat. ob-va [Proc. Moscow Math. Soc.], 1983, 46, 69-98 (in Russian).

8. V. V. Zhikov, "Spektralnyi podkhod k asimptoticheskim zadacham diffuzii" [Spectral approach to asymptotic diffusion problems], Diff. uravn. [Differ. Equ.], 1989, 25, No. 1, 44-50 (in Russian).

9. V.V. Zhikov, S.M. Kozlov, and O. A. Oleinik, Usrednenie differentsialnykh operatorov [Averaging of Differential Operators], Nauka, Moscow, 1993 (in Russian).

10. V.V. Zhikov and S.E. Pastukhova, "Ob operatornykh otsenkakh $\mathrm{v}$ teorii usredneniia" [On operator estimates in the theory of averaging], Usp. mat. nauk [Progr. Math. Sci.], 2016, 71, No. 3, 27-122 (in Russian).

11. T. Kato, Teoriia vozmushchenii lineinykh operatorov [Perturbation Theory for Linear Operators], Mir, Moscow, 1972 (Russian translation).

12. D. Kinderlehrer and G. Stampacchia, Vvedenie v variatsionnye neravenstva $i$ ikh prilozheniia [An Introduction to Variational Inequalities and Their Applications], Mir, Moscow, 1983 (Russian translation).

13. V. B. Korotkov, Integralnye operatory [Integral Operators], Nauka, Novosibirsk, 1983 (in Russian).

14. O. A. Ladyzhenskaia and N. N. Uraltseva, Lineinye $i$ kvazilineinye uravneniia ellipticheskogo tipa [Linear and Quasilinear Equations of Elliptic Type], Nauka, Moscow, 1973 (in Russian).

15. S.E. Pastukhova, "Approksimatsii operatornoi eksponenty v periodicheskoi zadache diffuzii so snosom" [Approximations of operator exponential in a periodic diffusion problem with a drift], Mat. sb. [Math. Digest], 2013, 204, No. 2, 133-160 (in Russian).

16. E. V. Sevostianova, "Asimptoticheskoe razlozhenie resheniia ellipticheskogo uravneniia vtorogo poriadka s periodicheskimi bystro ostsilliruiushchimi koeffitsientami" [Asymptotic expansion of solution of a secondorder elliptic equation with periodic fast oscillating coefficients], Mat. sb. [Math. Digest], 1981, 115, No. 2, 204-222 (in Russian).

17. T. A. Suslina, "Ob usrednenii periodicheskikh parabolicheskikh sistem" [On averaging of periodic parabolic systems], Funkts. analiz i ego prilozh. [Funct. Anal. Appl.], 2004, 38, No. 4, 86-90 (in Russian).

18. W. Feller, Vvedenie v teoriiu veroiatnostei $i$ ee prilozheniia. T. 2 [An Introduction to Probability Theory and Its Applications. V. 2], Mir, Moscow, 1967 (Russian translation).

19. A. Bensousan, J.L. Lions, and G. Papanicolaou, Asymptotic Analysis for Periodic Structure, North Holland, Amsterdam, 1978.

20. J.H. Ortega and E. Zuazua, "Large time behavior in $\mathbb{R}^{d}$ for linear parabolic equations with periodic coefficients," Asymptot. Anal., 2000, 22, No. 1, 51-85.

21. S.E. Pastukhova, "Approximations of the exponential of an operator with periodic coefficients," J. Math. Sci. (N.Y.)., 2012, 181, No. 5, 668-700. 
22. S.E. Pastukhova and R. N. Tikhomirov, "Error estimates of homogenization in the Neumann boundary problem for an elliptic equation with multiscale coefficients," J. Math. Sci. (N.Y.)., 2016, 216, No. 2, $325-344$.

23. V.V. Zhikov and S.E. Pastukhova, "Estimates of homogenization for a parabolic equation with periodic coefficients," Russ. J. Math. Phys., 2006, 13, No. 2, 224-237.

24. V.V. Zhikov and S.E. Pastukhova, "Bloch principle for elliptic differential operators with periodic coefficients," Russ. J. Math. Phys., 2016, 23, No. 2, 257-277.

V. V. Zhikov

Vladimir State University

87 Gor'kogo st., 600000 Vladimir, Russia

S. E. Pastukhova

Moscow Technological University (MIREA)

78 Vernadskogo avenue, 119454 Moscow, Russia

E-mail: pas-sedyandex.ru 